\title{
A tool for local thickness determination and grain boundary characterization by CTEM and HRTEM techniques
}

\author{
Á.K. Kiss ${ }^{1,2}$, E. F. Rauch ${ }^{3}$, B. Pécz ${ }^{1,2}$, J. Szívós ${ }^{1,2}$, J.L. Lábár ${ }^{1 *}$ \\ ${ }^{1}$ Hungarian Academy of Sciences, Research Center for Natural Sciences, Institute for Technical
} Physics and Materials Science, Konkoly Thege M. út 29-33, H-1121 Budapest, Hungary

${ }^{2}$ Doctoral School of Molecular-and Nanotechnologies, University of Pannonia, Faculty of Information Technology, Egyetem u. 10., H-8200 Veszprém, Hungary

${ }^{3}$ SIMaP, Grenoble INP/CNRS, 1130 rue de la Piscine - BP 75 - F-38402 St Martin D'Heres, France

Keywords: thickness determination, grain boundary (GB), grain boundary plane, high resolution (HRTEM)

\footnotetext{
*Corresponding author: labar.janos@ttk.mta.hu
} 


\section{Abstract}

A new approach to the measurement of the local thickness and characterization of grain boundaries is presented. The method is embodied in a software tool that helps finding and setting sample orientations useful for high resolution transmission electron microscopy examination of grain boundaries in polycrystalline thin films. The novelty is the simultaneous treatment of the two neighboring grains and orienting both grains and the boundary plane simultaneously. The same metric matrix based formalism is used for all crystal systems. Input to the software tool includes orientation data for the grains in question, which is determined automatically for a large number of grains by the commercial ASTAR program. Our software also helps identifying grains in a noisy orientation map. The grain boundaries suitable for HRTEM examination are automatically identified by our software tool. Individual boundaries are selected manually for detailed HRTEM examination from the automatically identified set. Goniometer settings needed to observe the selected boundary in HRTEM are advised by the software. Operation is demonstrated on examples from the cubic and hexagonal crystal systems.

\section{Introduction}

Characterization of crystalline material in a transmission electron microscope (TEM) necessitates tilting the sample to well defined crystallographic orientations irrespective of the fact, if diffraction studies, conventional bright field (BF) or dark field (DF) imaging or high resolution (HR) imaging is applied. Systematic tilting operations are based on determining the orientation of the crystal with respect to the laboratory system and also calibrating the orientations of the tilting axes of the 
goniometer relative to the same laboratory system. Determination of the orientation can be based on convergent beam electron diffraction (CBED) patterns (Edington, 1975) or on electron diffraction patterns recorded with parallel nanobeams (NB). For example CBED based orientation determination is implemented in the ProcessDiffraction program (Lábár, 2005) and NB based orientation maps are measured with the scanning-diffraction method (Rauch et al., 2008). An example of a tilting tool that pre-calculates the tilting values needed to reach a desired crystal orientation is the K-space Navigator program that also implements the tilts automatically, using piezoelectric actuators of the goniometer (Duden et al., 2011). Many studies are published where such tilting is applied for the examination of nanocrystalline materials by high resolution techniques, e.g. the atomic arrangement was explored within the crystals and the shape of the nanocrystals was determined in order to understand their growing mechanism. The cornerstone of all these experiments was the investigation of a chosen crystalline in many discrete low index orientations (Jinschek et al., 2008, Van Aert et al., 2011, Gontard et al., 2008, Habas et al., 2007). The accurate computer-control of the sample holder by Duden et al. (Duden et al., 2011) allowed orienting individual crystallites of nanometer size with high accuracy (Habas et al., 2007). Thus their software navigates in the reciprocal space - while the operator stays in real space imaging mode during the tilting operation so he/she can keep the examined area stationary (by correcting for unwanted shifts if needed).

When grain boundaries are to be examined, the situation is more complex since three related entities must be oriented simultaneously: both grains and the boundary plane between them. The software tool that we report here offers a solution to that more complex problem. 
The macroscopic properties of a GB are described by 5 parameters ( 5 degrees of freedom) namely the 3 parameters giving the relative crystallographic difference between their orientations (i.e. misorientation) and the 2 parameters describing the orientation (normal vector) of the crystallographic plane of the grain boundary (GB) between them (Randle, 1993). In that description even curved GBs are approximated locally by planes. That approximation is valid even for GBs with significant curvature (Forwood \& Clarebroug, 1991). The orientation of any individual crystallite means the relation between its native crystallographic system and the laboratory system i.e. corresponds to a coordinate-transformation that can be represented by an orientationmatrix. The misorientation between two grains is defined as a rotation transformation between the two Cartesian coordinate systems attached to the native crystallographic systems of the neighboring grains so it is deduced from the orientation matrices. The coincidence site lattice theory (CSL-theory) describes the misorientation of the neighboring grains for special low energy arrangements (Grimmer et al., 1974) but does not specify the orientation of the boundary plane between them.

Determination of the orientation of the boundary plane is more tedious than determination of the orientations of crystallites (grains). Such studies are frequently carried out in scanning electron microscopes (SEM) using electron backscatter diffraction (EBSD). In the simplest version of that method an orientation map of the grains is recorded with EBSD and direction of the surface line traces of GBs are identified. Since an infinite number of GB orientations can result in the same surface line trace, the method gives an incomplete characterization of the GB (Saylor et al., 2004). However, measuring only the orientation of the line trace of the GB-plane can be applied to decide whether the GB can be a special one or not (e.g. in case of $\Sigma 3$ misorientation: can the GB be $\{111\}$-like or not.) (Randle, 2001). Complete 
characterization applies a tedious 3-dimensional (3D) EBSD approach. First an EBSD map is recorded then a very thin layer of fixed thickness is removed (parallel to the original surface). These two steps are cycled and the 3D distribution of boundaries is reconstructed from following the virtual shift of the GB line traces as a function of depth (Saylor et al., 2003).

In this paper we show complete characterization of GBs with a semi-automatic procedure in the TEM. Although not completely automatic and concentrates only on a small number of boundaries at a time, our approach is easier than the one applying the 3D EBSD / FIB technique and provides a more complex operation than the methods orienting a single grain only. Subsequent to the macroscopic characterization we can also investigate selected GBs with HRTEM.

Our approach determines the orientation of the GB plane from the projected image of the polycrystalline thin film in the TEM. Whenever the GB plane is oriented oblique to the electron beam both the upper and the bottom line traces of the GB plane are discerned in the projected image and using the known local thickness of the thin film the elevation of the GB plane can be deduced. Local thickness can be determined from CBED for thicker crystalline samples (Kelly, 1975) or from comparing simulated HRTEM images to experimental ones (Stadelmann, 1987) in case of the thinnest films. As an alternative, the thickness of the sample can also be determined by electron energy loss spectrometry (EELS) (Egerton, 2011). Although the upper and lower traces can be told apart from the variation of the contrast for special GBs (Edington, 1976) in thicker films, we apply the more general method of stereographic reconstruction from a couple projections recorded with different tilts. The thickness of the sample was a necessary input parameter in our previous measurements (Lábár et al., 2012, Kiss et al., 2013), while the thickness is one of the variables determined as 
an integral part of our recent method presented in this paper. We only take the crystalline phase into account here, while any contamination or amorphous supporting layer has no impact on our thickness determination in contrast to the thickness value obtained by the EELS method.

However, for HRTEM the investigated area of the sample must be clean i.e. free of any contamination and must not be thicker than a few tens of nanometers. Furthermore, the investigated crystal have to be well oriented, the electron beam is only allowed to be parallel to low-index crystallographic directions. A manual orientation exercise based on diffraction requires practice from the TEM-operator. During tilting the sample, the investigated area may go out of focus and can also slide out of the viewing range. Therefore the final tilt position is usually reached after many tilt and correction cycles. During that lengthy procedure the illuminated area may be contaminated and become useless for HRTEM (Egerton et al., 2004). That is why the computer aided tilt procedure is needed.

Characterization is performed in three steps in our approach. First an orientation map is collected in the TEM with ASTAR (Rauch et al., 2008, http://www.nanomegas.com/). Grains with lateral size of > $20 \mathrm{~nm}$ can be mapped in our $\mathrm{LaB}_{6}$ TEM and many grains are evaluated in a single map. (Probe size and consequently the measurable grain size are limited by the brightness of our TEM and the need that a grain must extend several pixels.) Having an orientation map for many grains simultaneously is particularly important for finding GBs for HRTEM, since only a low fraction of all GBs are oriented in a direction that facilitates tilting the GB plane parallel to the electron beam due to the limited tilting range of the goniometer. 


\section{Steps of the procedure}

\section{Calibration of the microscope}

Pre-calculation of the needed tilt values in a double tilt holder needs two calibrations.

a) Rotation of the diffraction pattern relative to the image must be calibrated as a function of magnifications and the camera lengths used (Loretto \& Smallman, 1975).

b) In order to control the sample holder properly, the directions of its tilt-axes have also to be known in the laboratory system. These can be measured e.g. by studying the shift of a convergent beam electron diffraction (CBED) pattern while tilting the sample. The line trace of motion of the image of an arbitrary zone axis (the crossing point of Kikuchi-bands) assigns clearly the plane of the tilt, thus the axis of the rotation.

\section{Characterization of the GB plane from its projection and calculation of the local thickness in the TEM}

The orientation of a GB-plane is given by its normal vector. That normal vector (expressed in the laboratory system), and also the local thickness of the sample can be determined from either bright-field (BF) images (for thicker samples) or form HR images (for thin samples) of the GB. Both the width and the direction of the projection of the GB-plane have to be measured on BF images (Fig. 1), while the corresponding tilt-values of the double-tilt sample holder (goniometer) are input parameters of the calculation. Fig. 2 shows a schematic cross section of the sample 
containing a GB. All the notations used in this paper are consistent with the ones marked in the Fig. 1 and Fig. 2.

The laboratory-coordinate-system (image-system) is defined as follows: $\boldsymbol{X}$-direction: lies in the image plane, pointing right. $\boldsymbol{Y}$-direction: lies in the image plane, pointing upward. $Z$-direction: points perpendicular to the image plane, against the reader.

$\boldsymbol{d}$ - Width of the projection of the GB.

$\boldsymbol{\delta}$ - The angle indicating the direction of the projection of the GB in the image plane. This angle is measured from the X-direction in positive manner. $\delta=0 \ldots 180^{\circ}$

$\underline{\boldsymbol{n}}_{\text {trace }}$ - Unit vector, which is normal to the projection of the GB. It lies in the image plane. Its components are calculated with the help of $\boldsymbol{d}$ and $\boldsymbol{\delta}$.

The cross section of the sample described in Fig. 2 (i.e. the plane of the figure) lies perpendicular to the image plane and also perpendicular to the projection of the GB (i.e. parallel to $\underline{\boldsymbol{n}}_{\text {trace }}$ ).

$\boldsymbol{b}$ - The part of the GB lying in the plane of the cross-section indicated in Fig. 2.

$\underline{\boldsymbol{n}}_{\mathrm{GB}}-$ Unit vector, normal to the plane of the GB.

$t$-Thickness of the sample.

$\boldsymbol{\omega}$ - The angle between the sample-surface and the GB-plane. $\boldsymbol{\omega}=0^{\circ} \ldots 180^{\circ}$

Since either the width or the direction of the projection of a GB do not change while tilting the sample about an axis lying parallel to $\underline{\boldsymbol{n}}_{\text {trace, }}$, the tilt positions of the sample differing only in a rotation component about this axis are experimentally equivalent. Therefore we consider all the tilt positions as they were tilted only about an axis parallel to the projection of the GB. This is how $\boldsymbol{\alpha}$ can be calculated: 
$\boldsymbol{\alpha}$ - the angle between the sample-surface and the image plane in the aforementioned manner. $\boldsymbol{\alpha}=-90^{\circ} \ldots+90^{\circ}$ If $\boldsymbol{\beta}$ is the angle between $\underline{\boldsymbol{n}}_{\text {trace }}$ and the normal direction of the sample, then:

$$
\alpha=90^{\circ}-\beta
$$

At the zero-tilt position the normal direction of the sample-surface corresponds to the unit vector pointing in the $Z$-direction. Since the directions of the tilt axes of the sample-holder are known, the normal of the sample-surface can be easily calculated.

According to Fig. 2, the following equations can be written with the variables of $t$ and $\omega$ :

$$
\left\{\begin{array}{c}
\cos (\alpha+\omega)=\frac{d}{b} \\
\sin (\omega)=\frac{t}{b}
\end{array} \rightarrow \mathrm{t} \cdot \cos (\alpha+\omega)=d \cdot \sin (\omega)\right.
$$

Therefore the following equation system has to be solved for $\boldsymbol{t}$ and $\boldsymbol{\omega}$, where the input parameters $\boldsymbol{d}_{1}, \boldsymbol{\alpha}_{1}$ and $\boldsymbol{d}_{2}, \boldsymbol{\alpha}_{2}$ come from measurements based on two different tilt positions:

$$
\left\{\begin{array}{l}
\mathrm{t} \cdot \cos \left(\alpha_{1}+\omega\right)=d_{1} \cdot \sin (\omega) \\
\mathrm{t} \cdot \cos \left(\alpha_{2}+\omega\right)=d_{2} \cdot \sin (\omega)
\end{array}\right.
$$

Thus (here $\boldsymbol{t}$ can be expressed both with $\boldsymbol{d}_{1}, \boldsymbol{\alpha}_{1}$ or $\boldsymbol{d}_{2}, \boldsymbol{\alpha}_{2}$ ):

$$
\cot (\omega)=\frac{d_{1} \sin \left(\alpha_{2}\right)-d_{2} \sin \left(\alpha_{1}\right)}{d_{1} \cos \left(\alpha_{2}\right)-d_{2} \cos \left(\alpha_{1}\right)}
$$


Thickness determination and GB examination in polycrystalline samples

$$
t=\frac{d_{1 \mid 2}}{\cot (\omega) \cdot \cos \left(\alpha_{1 \mid 2}\right)-\sin \left(\alpha_{1 \mid 2}\right)}
$$

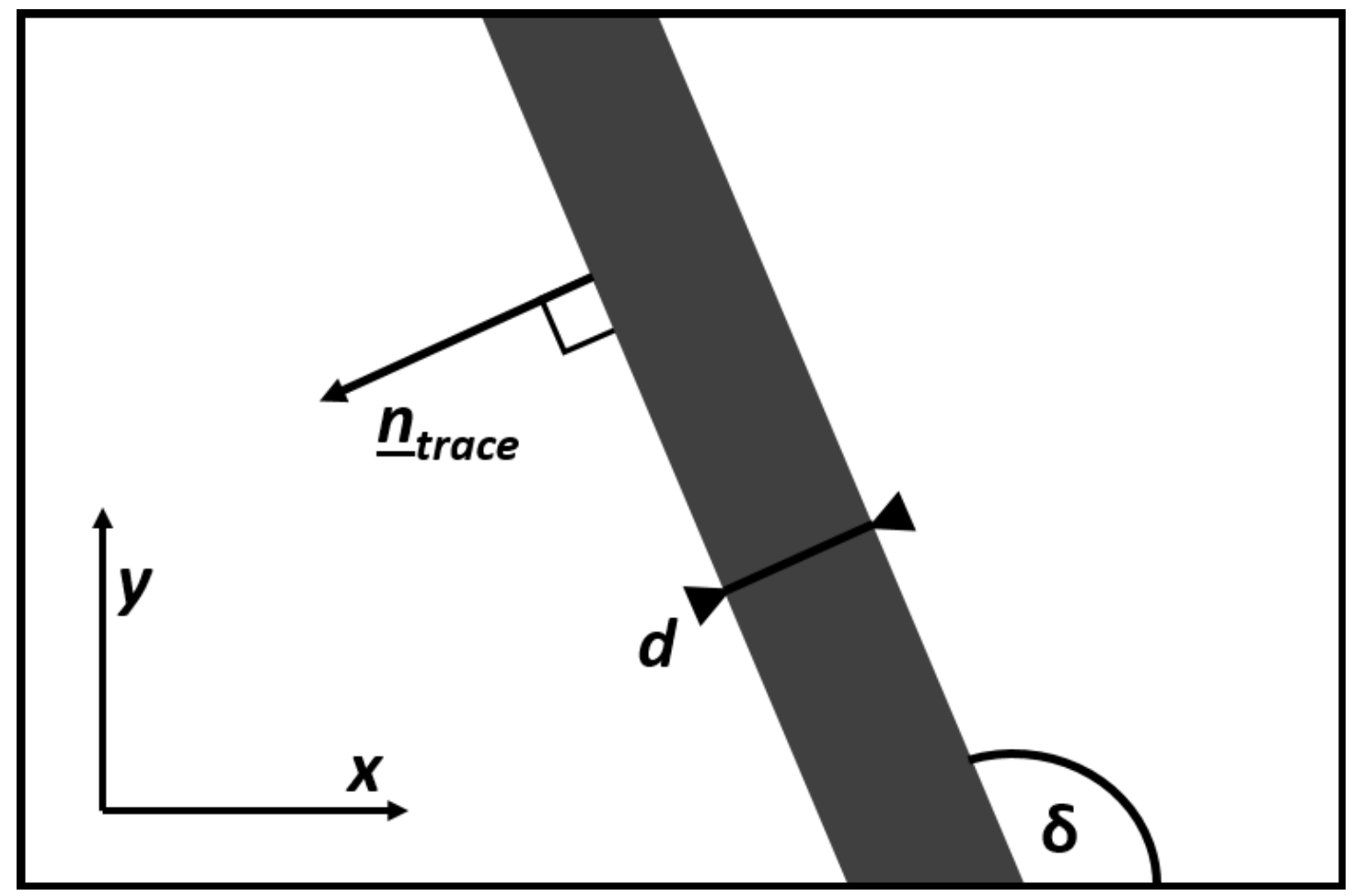

Fig. 1. Schematic illustration of the projection of a GB in the image. The trace is illustrated by the gray band its width and direction are characterized by $d$ and $\delta$, respectively. The normal direction of the projection $\left(\underline{\boldsymbol{n}}_{\text {trace }}\right)$ is expressed by these measured values. 


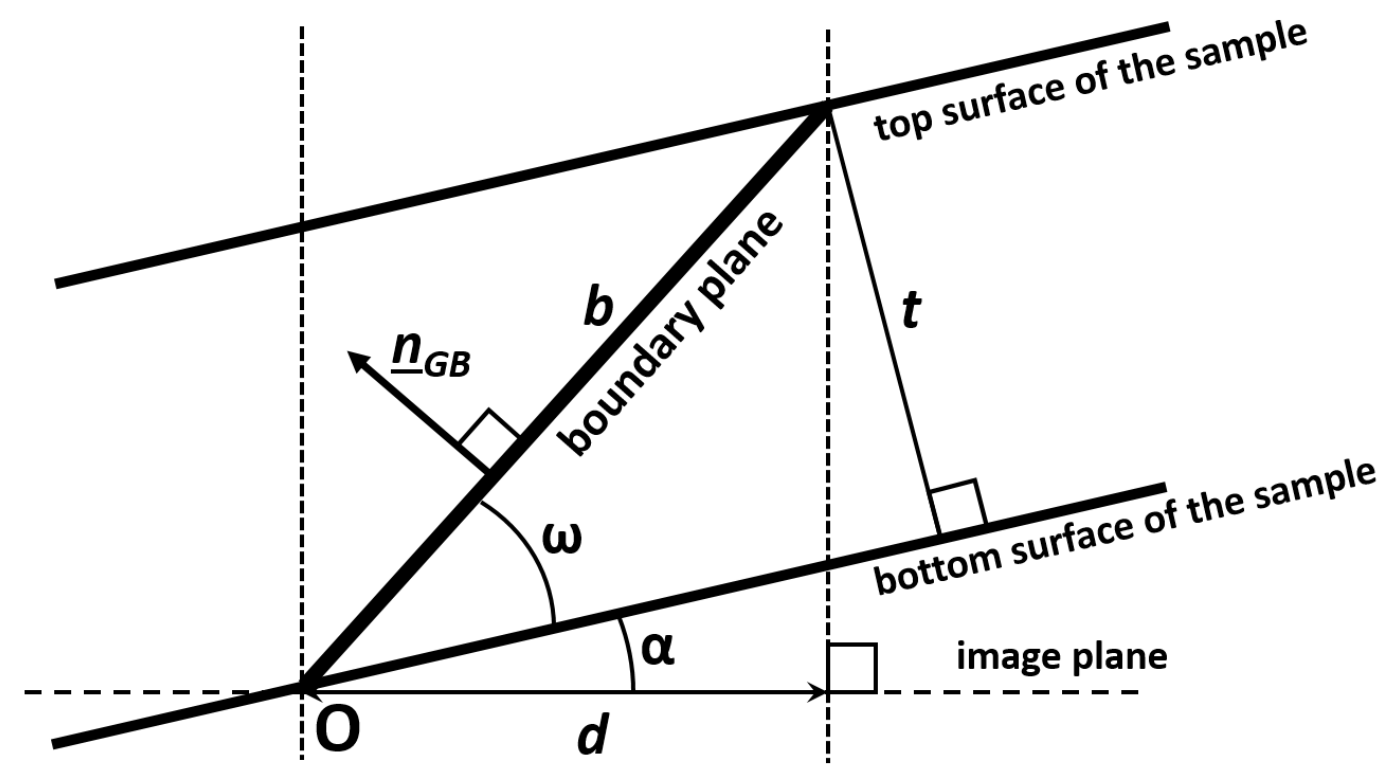

Fig. 2 Schematic cross section of a TEM-sample containing a grain boundary.

Fig. 3 shows, that both a right and a left tilted GB can produce the same projection in the image plane. If it is not identified, which edge of the projection belongs to the upper and which to the lower surface of the sample, due to this ambiguity, the value of $\cos (\alpha+\omega)$ in (eq. 1) can be either positive or negative. Therefore the measured widths of the projections have to be tested with both positive and negative sign of $\cos (\alpha+\omega)$. Thus (eq. 2) have to be evaluated in four ways, since we have no prediction about that, what sign of $\cos (\alpha+\omega)$ the measured $d$ values have to be substituted with. Two ${ }^{1}$ of the four solutions describe physically possible cases resulting in positive values for the sample thickness. These solutions are illustrated in Fig. 4. In practice, if one solution for the local thickness can be rejected, because it can physically be ruled out (e.g. so thick that it could not be

${ }^{1}$ According to (eq. 3) and (eq. 4), when both $\boldsymbol{d}_{\boldsymbol{l}}$ and $\boldsymbol{d}_{2}$ change sign, $\boldsymbol{c o t}(\boldsymbol{\omega})$ will not change and $\boldsymbol{t}$ will change sign. Therefore the four solutions for $t$ contains two pairs, each with equal absolute values but with positive and negative sign. 
transmitted by the electrons) then only one solution for $t$ is left, thus the elevation of the GB-plane is determined unambiguously. If it is not, an additional measurement with other tilt settings is needed: by evaluating (eq. 4) with the possible input data pairs, the solution, common to the two data-pairs select the physical relevant values for local thickness and the corresponding GB-plane. When the normal vector of the GB is expressed in the laboratory system, the corresponding $h k l$ indices of the plane can be expressed in both native-systems of the neighboring grains with the help of a linear transformation using the two orientation matrices (see following chapter).

To improve the accuracy of the measurements it is recommended to choose such a goniometer setting in which the projection of the GB can be measured easily. In the case of a relatively thick sample, one of the neighboring grains may be oriented in two-beam condition: in this case there are thickness fringes ${ }^{2}$ in the projection of the boundary result is sharp contrast at the edges of the projection (Fig. 5/a). When the sample is prepared for HRTEM imaging, the thickness is too small for producing such thickness fringes. In this case HRTEM can be used (see following chapters): since the superposition of the neighboring line-periodicities appears in the overlapping area, the edges of the projection can be measured with a good accuracy (Fig. 5/b).

\footnotetext{
${ }^{2}$ The thickness fringes appear due to the wedge shaped part of the crystal cut by the grain boundary.
} 


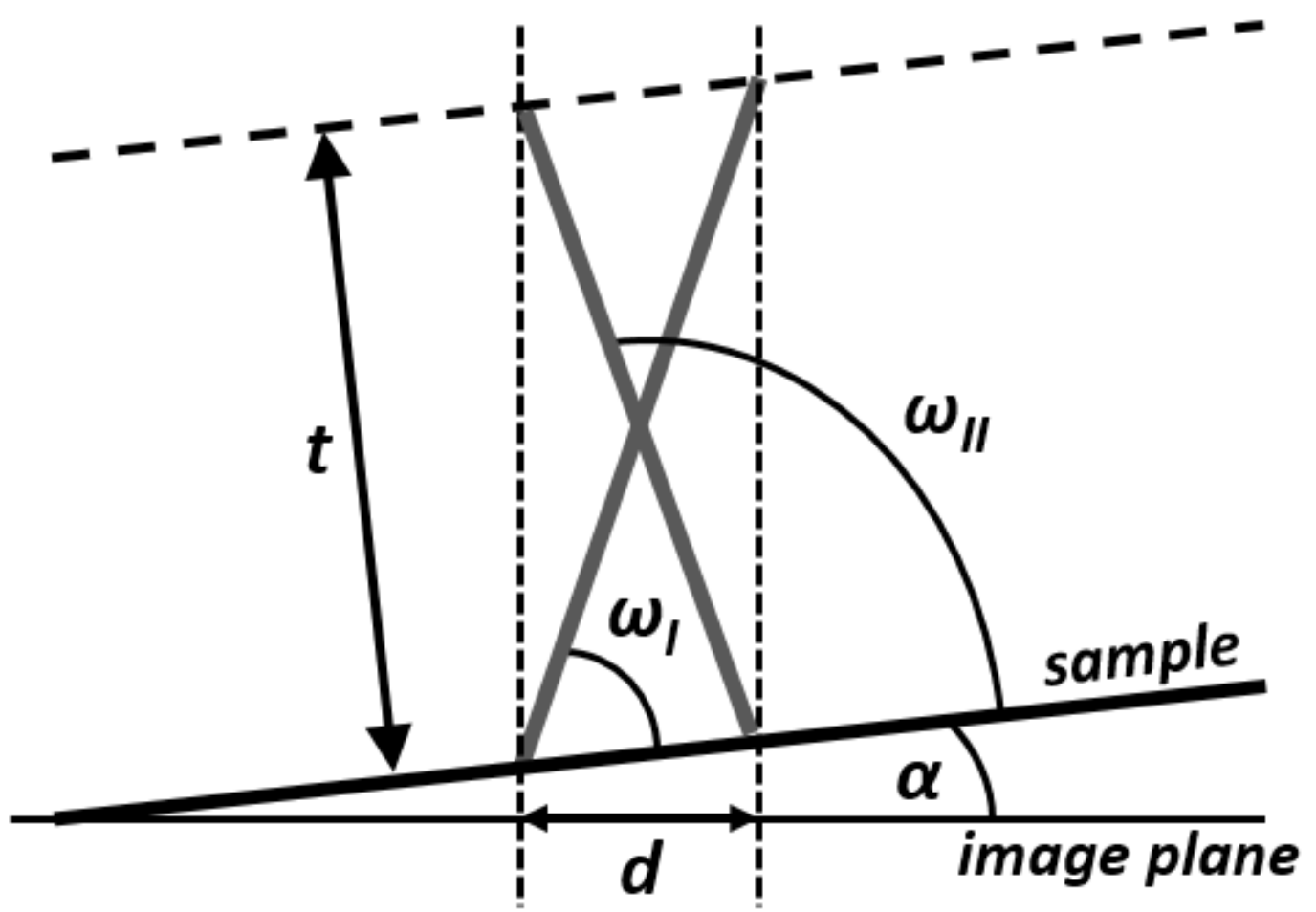

Fig. 3. Schematic illustration of the fact that both the right and the left tilted GBs (marked by thick gray lines) can produce the same projection in the image plane.
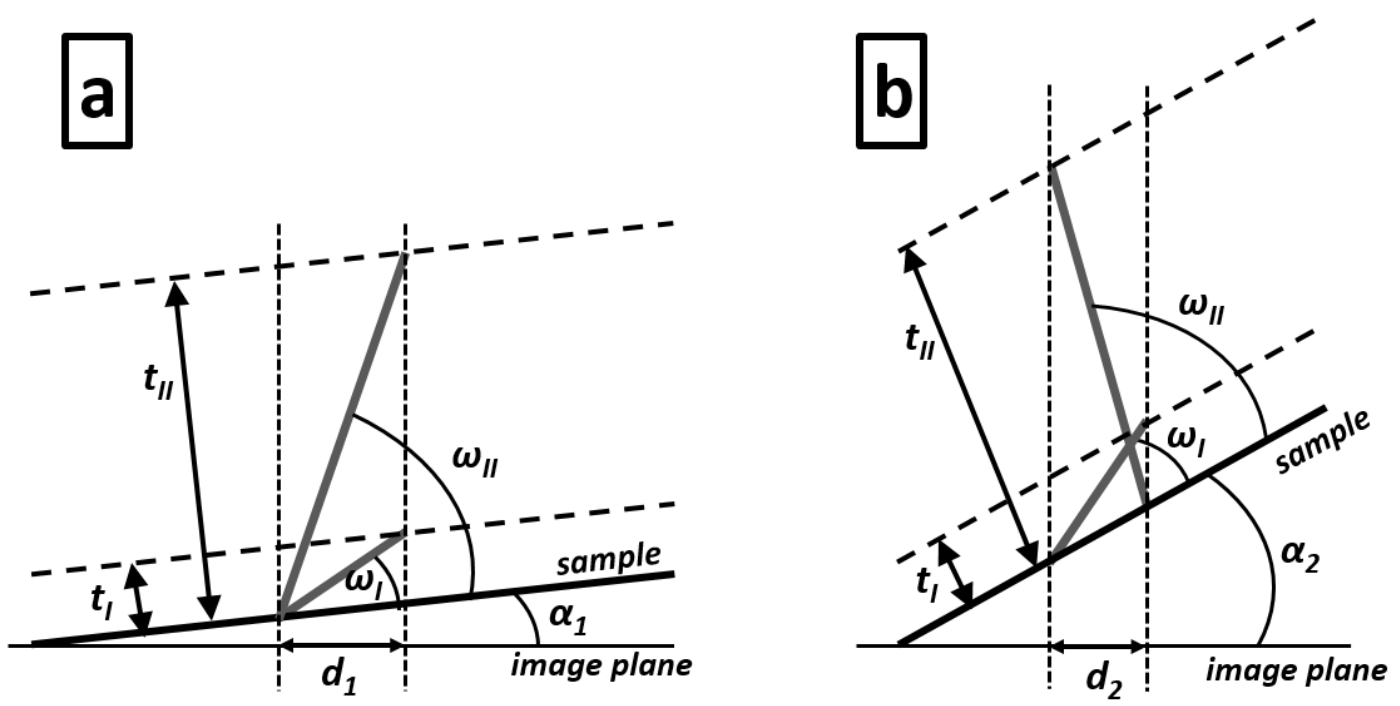

Fig. 4. Schematic illustration of the tilt ambiguity of the GB-plane. Both of the two kinds of samples (one with the thickness $\boldsymbol{t}_{I}$ and the other with $\boldsymbol{t}_{I I}$ ) produces the same 
projection in each of the two tilt positions $(a),(b)$. Note that the elevation $\left(\omega_{I}, \omega_{I I}\right)$ of the theoretical GBs are the same, constant in both (a) and (b).
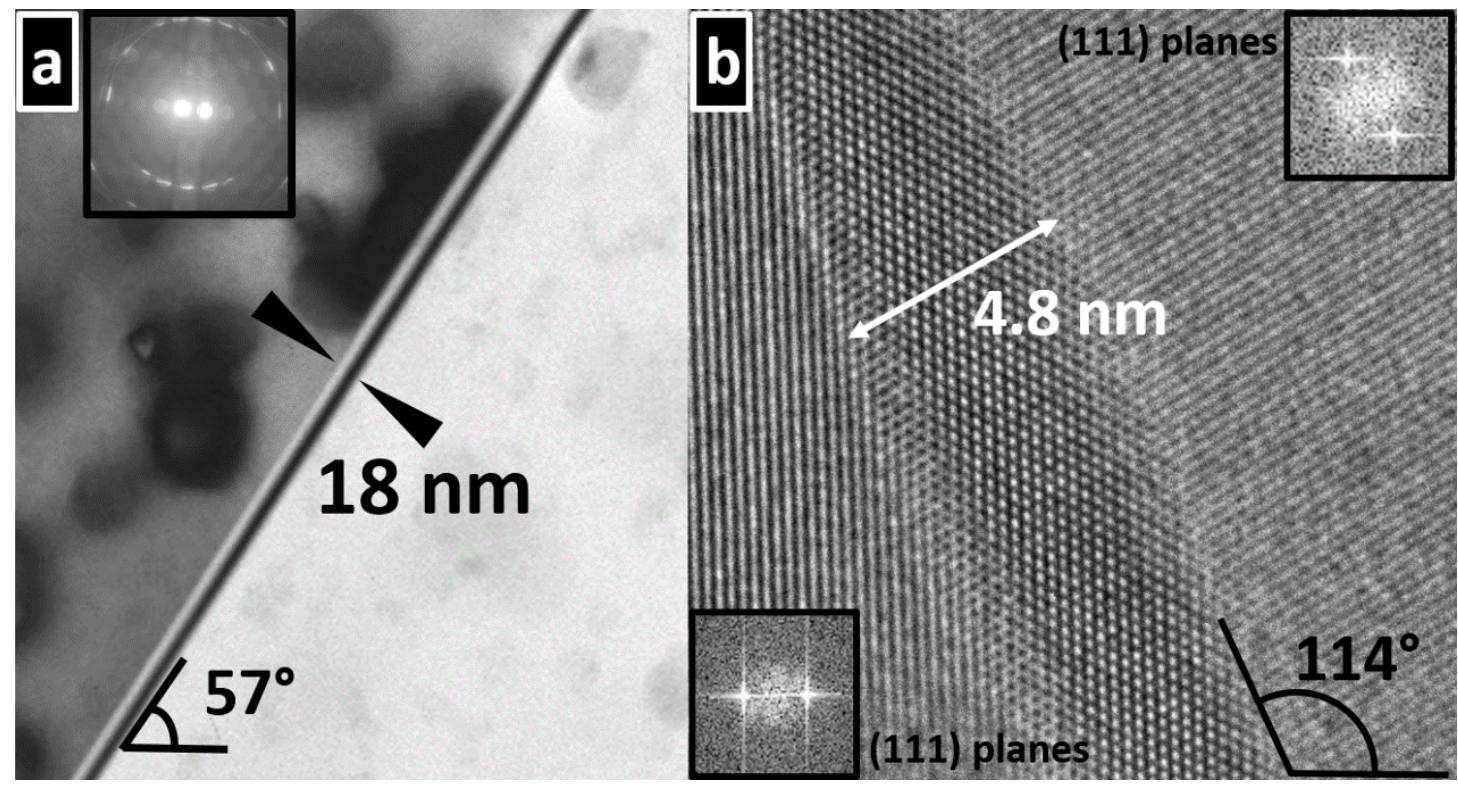

Fig. 5. Bright field image taken on polycrystalline Si thin film (a) and HR image taken on polycrystalline Al film (b). The azimuth angles and the width are marked. The sharp contrasts of the projected GBs are significant. In the image (a), the grain on the left hand side is oriented in two beam condition therefore the thickness fringes are seen very well. In image (b), both of the neighboring grains are imaged with plane resolution, resulting in well observable pattern in the overlapping area. Due to the misorientation angle of $60^{\circ}$ between the planes of the two grains by chance, the Moiré pattern and a simple superposition look the same.

\section{Measurement of orientation, orientation mapping}

Evaluating a Kikuchi-band map on a CBED pattern is one of the most accurate methods of the local measurement of orientation and we started by testing that method manually on a selection of grains. However, for manual CBED measurements the 
local sample thickness has to be thicker than optimal for HRTEM. Additionally, measuring the grains one-by-one is very time-consuming; thus this method does not satisfy our need to collect many orientation data from a large area in reasonable time. That is why the orientation maps were finally measured with the commercial Astar scanning/precession tool (Rauch et al., 2008, http://www.nanomegas.com/). installed on a JEOL 3010 HRTEM. That tool provides an orientation map from the area of interest with reasonable accuracy in a quite short time (tens of minutes). Every pixel in the orientation map contains local orientation and phase information about the corresponding location of the sample, i.e. the three Euler-angles and a phase-index are stored in each pixel. Since in the next step we use the orientation matrix formalism, the Euler angles are converted into orientation matrices.

The orientation matrix $(O)$ describes a direct connection between the directioncoordinates expressed in the laboratory-system and in the native crystallographic system:

$$
r_{\text {Lab }}=O \cdot r_{\text {nativ }}
$$

The $\mathrm{O}$ orientation matrix can be written in the following way.

$$
O=N^{-1} \cdot M
$$

The $N$ matrix is calculated from the $\varphi, \theta, \psi$ Euler-angles and gives the relation between the laboratory-system and the virtual Cartesian-system attached to the native crystal-system. Thus $N$ matrix characterizes only the rotation of the grain compared to the laboratory system without having any information about the crystal-structure. $N$ can be expressed by the Euler-angles (Randle, 1993): 


$$
\begin{aligned}
& N_{0,0}=\cos \varphi \cos \psi-\sin \varphi \sin \psi \cos \theta \\
& N_{0,1}=\sin \varphi \cos \psi+\cos \varphi \sin \psi \cos \theta \\
& N_{0,2}=\sin \psi \sin \theta \\
& N_{1,0}=-\cos \varphi \sin \psi-\sin \varphi \cos \psi \cos \theta \\
& N_{1,1}=-\sin \varphi \sin \psi+\cos \varphi \cos \psi \cos \theta \\
& N_{1,2}=\cos \psi \sin \theta \\
& N_{2,0}=\sin \varphi \sin \theta \\
& N_{2,1}=-\cos \varphi \sin \theta \\
& N_{2,2}=\cos \theta
\end{aligned}
$$

The $M$ matrix describes the relation between the native crystallographic-system and the Cartesian-system attached to the grain. The $M$ matrix is expressed by the native cell-parameters:

$$
\begin{aligned}
& M_{0,0}=a \\
& M_{0,1}=0 \\
& M_{0,2}=0 \\
& M_{1,0}=b \cdot \cos \gamma \\
& M_{1,1}=b \cdot \sin \gamma \\
& M_{1,2}=0 \\
& M_{2,0}=c \cdot \cos \beta \\
& M_{2,1}=c \cdot \frac{\cos \alpha-\cos \beta \cos \gamma}{\sin \gamma} \\
& M_{2,2}=c \cdot \frac{\sqrt{\left(1-\cos ^{2} \alpha-\cos ^{2} \beta-\cos ^{2} \gamma+2 \cos \alpha \cos \beta \cos \gamma\right)}}{\sin \gamma}
\end{aligned}
$$

Here $a, b, c$ are the lengths of the lattice-vectors, $\alpha, \beta, \gamma$ are the opposite angles of the unit cell. The $x, y, z$ Cartesian-system attached to the native system is created following one of the usual crystallographic conventions (International tables for crystallography Vol. B, 2001) (Fig. 6): 


$$
x \| a
$$

$y \perp_{x}$ and lies in the plane defined by $a, b ;$

$$
z \| c^{*}
$$

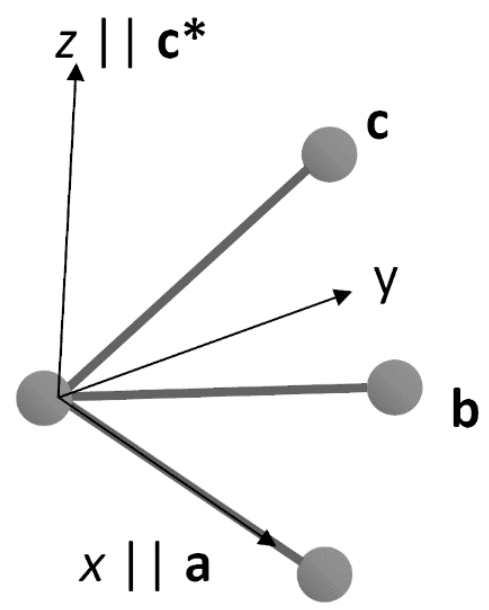

Fig. 6. The relation between the native crystallographic-system and the attached Cartesian-system.

\section{Automatic identification of the grains; noise reduction}

The primary input to our software that identifies grains and grain boundaries is a pixel-by-pixel orientation map (Fig. 7/a). By visual inspection of the colored orientation map we have a rough idea about the extension of the grains. However, due to noise and the occasionally low reliability of indexing it is not perfectly obvious which pixels belong to a grain and which pixels belong to the next grain and which ones to the GB between them. Our software first classifies each pixel to yield grain areas with (a common, approximately) homogeneous orientations and GB areas between them. 
Classification is based on the fact that at least one Euler angle must change significantly as a GB is crossed. So, we display the three Euler-angles in three separate images where each pixel contains the corresponding value of displayed Euler-angle. Then we apply the "Sobel" edge-detection method (Sobel, I., 1978, Qu Ying-Donga et al., 2005) on each of the three images one-by-one. As a result, in all three images the procedure highlights the pixels that are situated in a region where the displayed Euler angle changes significantly. The edges, detected on these 3 images are combined, resulting in a single GB-map. The contrast in the GB map is related to the angular change across the GB. This means that the high-angle boundaries appear with stronger contrast than the low-angle boundaries. If we do not want to subclassify the GBs, only detect them then all edges above a threshold intensity level are accepted as GB pixels. The pixels with lower (than the threshold) contrast will be set white and the pixels with higher contrast will be set black, therefore the noise within the grains can be filtered out and in the case of a low threshold value both the lowangle boundaries and the high-angle boundaries are identically highlighted. If the threshold is increased to about $15^{\circ}$ only high angle boundaries are identified.

Next the grains in this black-and-white GB-map are given unique sequence numbers. All the white pixels that belong to the same grain get the same unique sequence number (Fig. 7/b). Referring to each grain by its own sequence number, both a phase index and an average orientation will be assigned to each grain. The average orientation is calculated by averaging the Euler-values stored in the grain's pixels. Thus unique orientation matrices are assigned to each grain. Next, all data belonging to both neighboring grains are stored for the pixels of the GB between them (Fig. 7/c). That approach must be useful in characterizing either grain boundaries or 
phase boundaries. (Obviously, this approach is not intended to be used to characterize in-grain curvature, twist, etc.)

Next the average orientation data of the grains are compared to the orientations of each of their neighbors: in case of finding appropriate pairs, the GB-pixels containing the same neighbor-grain sequence numbers will be highlighted.

For the favorable case when both grains can be tilted to zone axis direction for examination, the tilt-angles needed to orient those zones in beam-direction are calculated for those pairs, which are simultaneously within the tilting range of the sample holder. That procedure is straightforward. The question still remains if any of these zone axis pairs lie parallel to the respective GB plane.

In the worst case scenario when only one plane per grain can be resolved we find the needed common orientation with a different algorithm. We find all the $h k l$ indexes, which are representing resolvable sets of crystal planes. First the real space direction $[\mathrm{nmp}]$ is found that is parallel to the $h k l$-directions of the reciprocal-space using the metric-tensor $(G)$ of the given crystal structure (Spence, 1992).

$$
\begin{gathered}
\left(\begin{array}{l}
n \\
m \\
p
\end{array}\right)=G^{-1} \cdot\left(\begin{array}{l}
h \\
k \\
l
\end{array}\right) \\
G=\left(\begin{array}{lll}
\mathbf{a} \cdot \mathbf{a} & \mathbf{a} \cdot \mathbf{b} & \mathbf{a} \cdot \mathbf{c} \\
\mathbf{b} \cdot \mathbf{a} & \mathbf{b} \cdot \mathbf{b} & \mathbf{b} \cdot \mathbf{c} \\
\mathbf{c} \cdot \mathbf{a} & \mathbf{c} \cdot \mathbf{b} & \mathbf{c} \cdot \mathbf{c}
\end{array}\right)
\end{gathered}
$$

Here $m, n, p$ are indices in direct-space, $h, k, l$ are indices in reciprocal-space (Miller-indices) and $\mathbf{a}, \mathbf{b}, \mathbf{c}$ are the base-vectors of the real space lattice. 
Now these normal vectors of planes (given in the direct-space native crystal system) are transformed into the laboratory system and we can build pairs where the first member of the pair comes from the first grain, the second one comes from the other. After the cross-product was calculated for a pair, the resulting direction may be tilted parallel to the electron beam. (Since the laboratory system is a Descartes system, parallel directions in real space and reciprocal space are characterized with the same indices.) From that direction we can take an HRTEM image in which one plane per grain is resolved simultaneously.

Our software searches automatically all neighboring grain pairs and highlights their boundary when they can be tilted for HRTEM taking into account the tilting limits of the sample holder. By double-clicking on a highlighted GB the accessible zones or planes are listed together with the needed tilt values. Subsequently the tilting operation is done in imaging mode and the grains are kept stationary by the operator.

It is important to note that both the identification of the orientation and the tilt operation is of finite accuracy. First, the orientation is determined from a searchmatch algorithm by ASTAR where the measured pattern is matched to a set of precalculated patterns (templates). Templates are usually calculated in $1^{\circ}$ steps. Additionally the accuracy of our goniometer is about $0.2^{\circ}$. Additionally, small orientation changes can also be present within the grains due to the presence of dislocations. As a consequence, fine-tuning of the tilt values are needed around the calculated tilt values as show in the examples section.

\section{Investigation of GBs by HRTEM}

In order to get a lattice resolution image on the chosen crystal with more than one lattice planes resolved simultaneously, the beam must be parallel to a low index 
zone axis direction (the number of available zones depends on the point resolution of the microscope). When these low-index zone axis directions are out of the tilting range of the sample holder, orienting only one set of resolvable planes parallel to the beam can still be possible and a single lattice plane can be imaged up to the line resolution of the HRTEM. When GBs are to be examined by HRTEM, preferably several lattice planes should be imaged in each of the grains simultaneously and the GB plane also should be set parallel to the electron beam at the same time. In the case of a random orientation distribution of grains, there is little chance to find parallel low-index zone axes in both neighboring grains, and the chance is even lower to find them within the tilting range of the sample holder. So, occasionally it is impossible to fulfill all these conditions simultaneously and no solution exists at all. In the least favorable case one plane per grain is only resolved simultaneously and the GB plane remains oblique to the electron beam. This last condition provides the minimal information about the GB structure. Of course, the looser the expected experimental conditions are, the more boundaries will be accessible for examination (Fig. 7/d,e,f). Any of the above HRTEM situations are difficult to find manually and we must be very lucky to find an appropriate pair of grains in a polycrystalline sample without the help of a computer.

Our software tool helps to find these appropriate GBs in a polycrystalline thin film and gives advice how to tilt the double-tilt sample holder in order to obtain high resolution images. First an orientation map is recorded with the help of the Astar scanning/precession tool installed on the HRTEM, and then our software processes the orientation map, also using the actual experimental conditions, such as the resolution limit of the microscope, the directions of the tilt axes in the laboratory system and the value of the maximal available tilt. On the resulting GB-map the GBs 
are highlighted that are available for HRTEM investigation. This process highly increases the efficiency of the investigation of GBs: many boundaries can be identified on the orientation map and can be evaluated in a short time; therefore we have a reasonable chance to find a few GBs which are appropriate to investigate them within our actual experimental conditions.

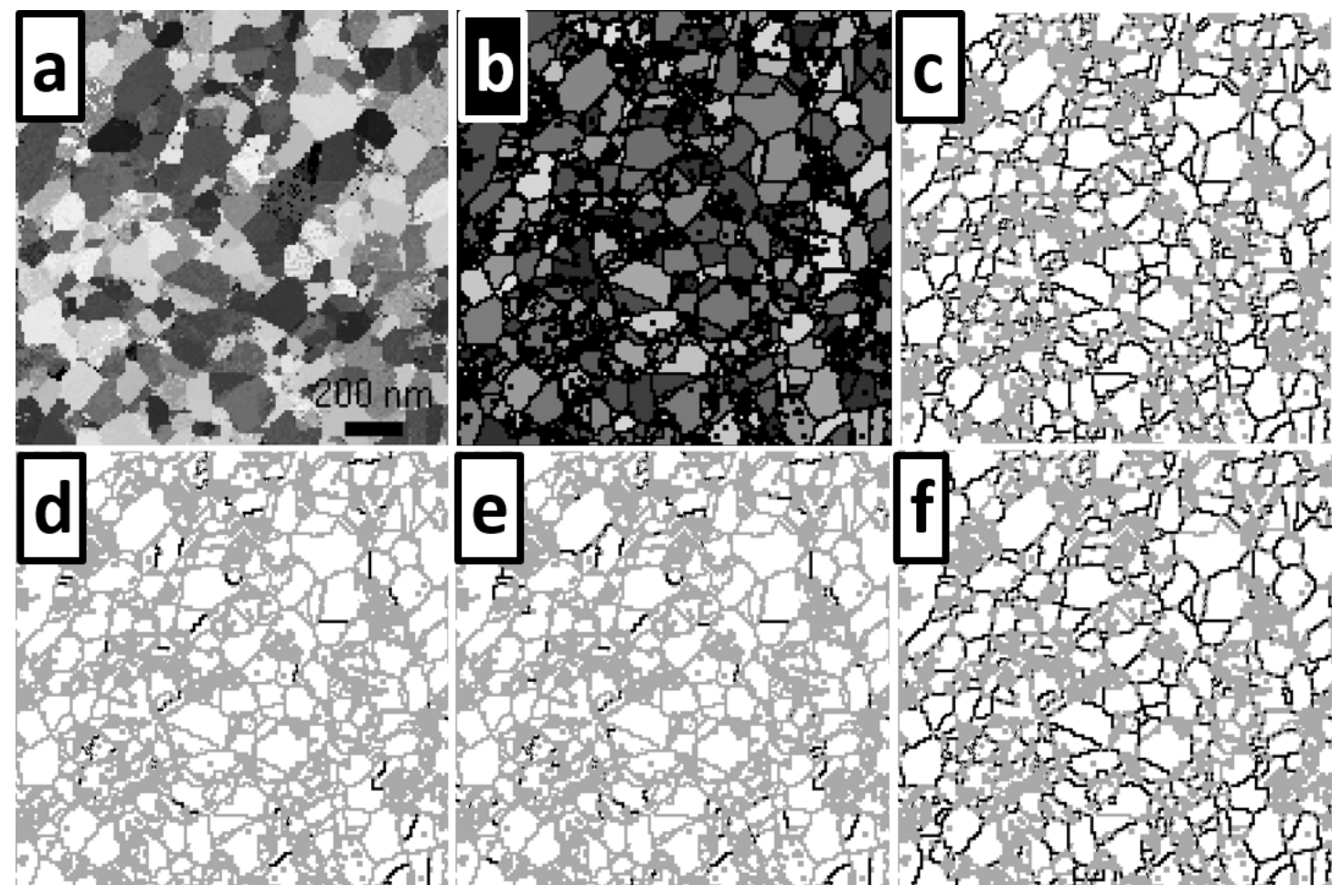

Fig. 7. The evaluation steps of an orientation map presented on polycrystalline

Al film. Orientation map (a), the single grains and the pixels which belong to GBs are identified (b), the part of the GBs which are appropriate for further calculations are displayed by black (c), the boundaries are highlighted by black, where the neighboring grains can be imaged by simultaneous lattice resolution (d), by simultaneous plane and lattice resolution (e) and by simultaneous plane resolution (f). 


\section{Experimental demonstration of use}

Application of our software tool is demonstrated on both a polycrystalline $f c c$ $\mathrm{Al}$, and on hcp $\mathrm{ZnO}$ sample. The self-supporting $\mathrm{Al}$ layer was gown by $\mathrm{DC}$ magnetron sputtering on thin amorphous carbon film supported by a copper TEM-grid. (The base pressure was $1.6 * 10^{-7}$ mbar and the argon pressure during deposition was $2.5^{*} 10^{-3}$ mbar. Aluminium was sputtered at $100 \mathrm{~W}$ power for $5 \mathrm{~min}$. The sample was annealed at $250^{\circ} \mathrm{C}$ in $1.4 * 10^{-7}$ mbar for $30 \mathrm{~min}$.) The investigations were made with a JEOL 3010 HRTEM equipped with an Astar scanning/precession device.

All the orientation maps presented in this paper are colored by the color code ${ }^{3}$ shown in Fig. 8 . One of the basic directions (X: points to the right, Y: points upward or Z: direction of observation, normal to the plane of the image) of the sample can be selected to color code the orientation distribution of that direction in the orientation map.

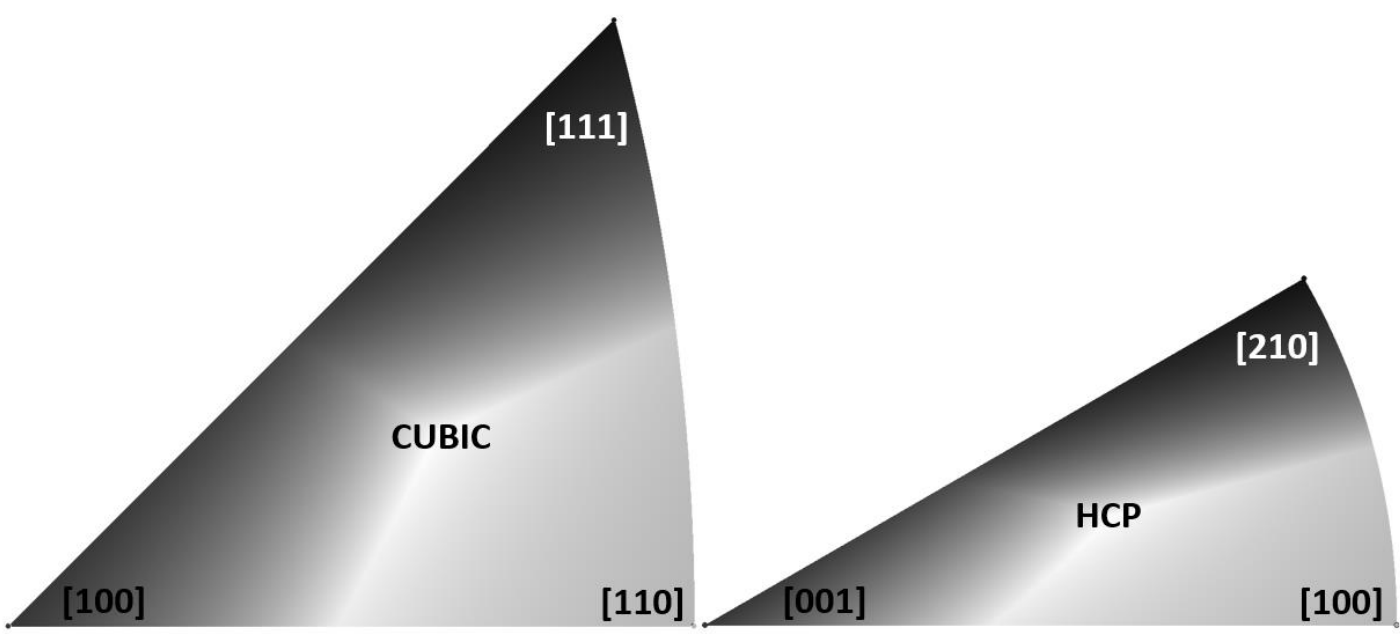

Fig. 8. Color coding in orientation maps for cubic and hexagonal structures.

\footnotetext{
${ }^{3}$ For readers of the B/W printed version, it is only important that the different grains in Fig. 7/a,
} Fig. 9/a, Fig. 13/a and Fig. 16 are shown with different shades. 


\section{Example I.}

In our first example the simplest case, a twin boundary with $\sum 3$ misorientation in a cubic material (Al) is shown. After an orientation map had been taken on the area of interest, the boundaries have been highlighted where both neighboring grains can be investigated in exact zone direction (Fig. 9, Fig. 10). The errors introduced by the evaluation led to a non-negligible inaccuracy in the calculated values compared to the refined experimental ones. The final tilt differs from the calculated tilt by $1-2^{\circ}$, and the calculated orientation deviation (OD) between the two expected zone directions achieves almost $1^{\circ}$ (Table 1). Since the $\sum 3$ misorientation predicts no OD, our calculated results can only be attributed to the evaluation errors which have been introduced by the inaccuracy of the orientation identification and by the bending of the sample.

The grain size is about $100 \mathrm{~nm}$ and the orientation map has been collected with $10 \mathrm{~nm}$ spot size. After the tilt refinement both neighboring grains are imaged in exact [110] zone directions (Fig. 11). The boundary plane has (111) characteristics (coherent twin boundary) with perpendicular (211) steps (incoherent twin boundary) (Fig. 12).

Table 1. Experimental conditions of HTEM imaging

\begin{tabular}{|l|l|l|l|l|}
\hline Grain & Zone to reach & $\begin{array}{l}\text { Predicted OD (here } \\
\text { it is an indication }\end{array}$ & Calculated \\
of calculation & tilt position & Refined tilt position \\
& error) & & axis orientation \\
\hline
\end{tabular}




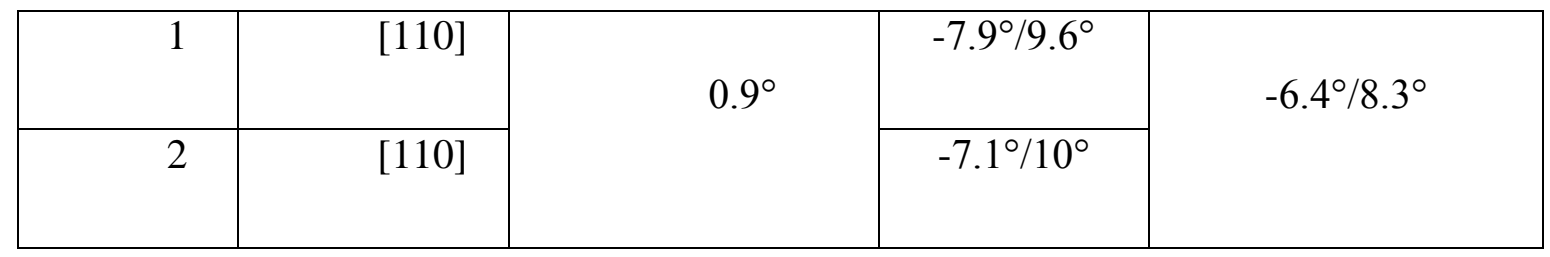

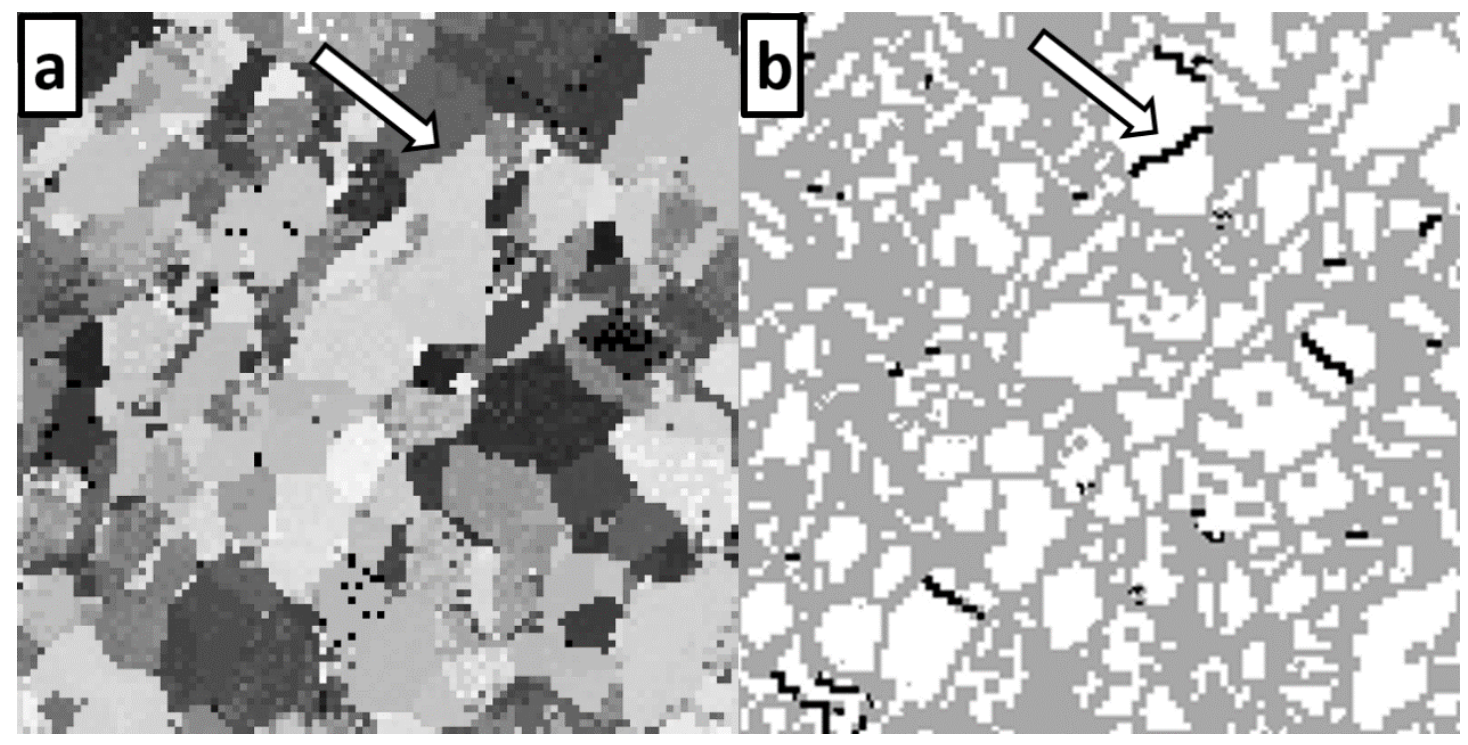

Fig. 9. Orientation map of an Al foil - the coloring (shading) displays the orientation distribution in the X-direction (a). The black-highlighted boundaries are available for HRTEM imaging (b). The investigated boundary is marked by an arrow. 


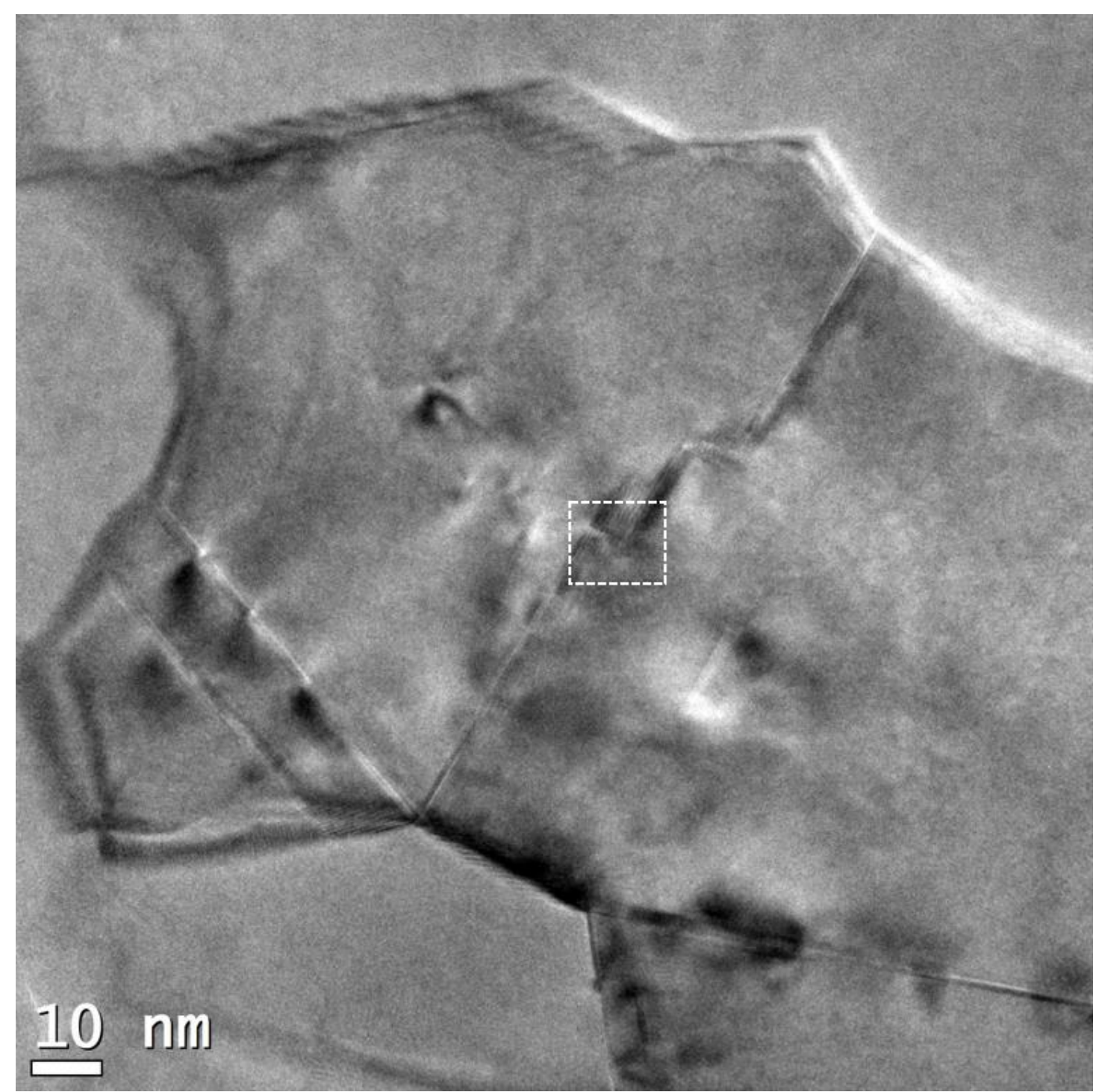

Fig. 10. The studied grains shown with low magnification. The part of the boundary investigated by high resolution is marked by the dashed rectangle. 


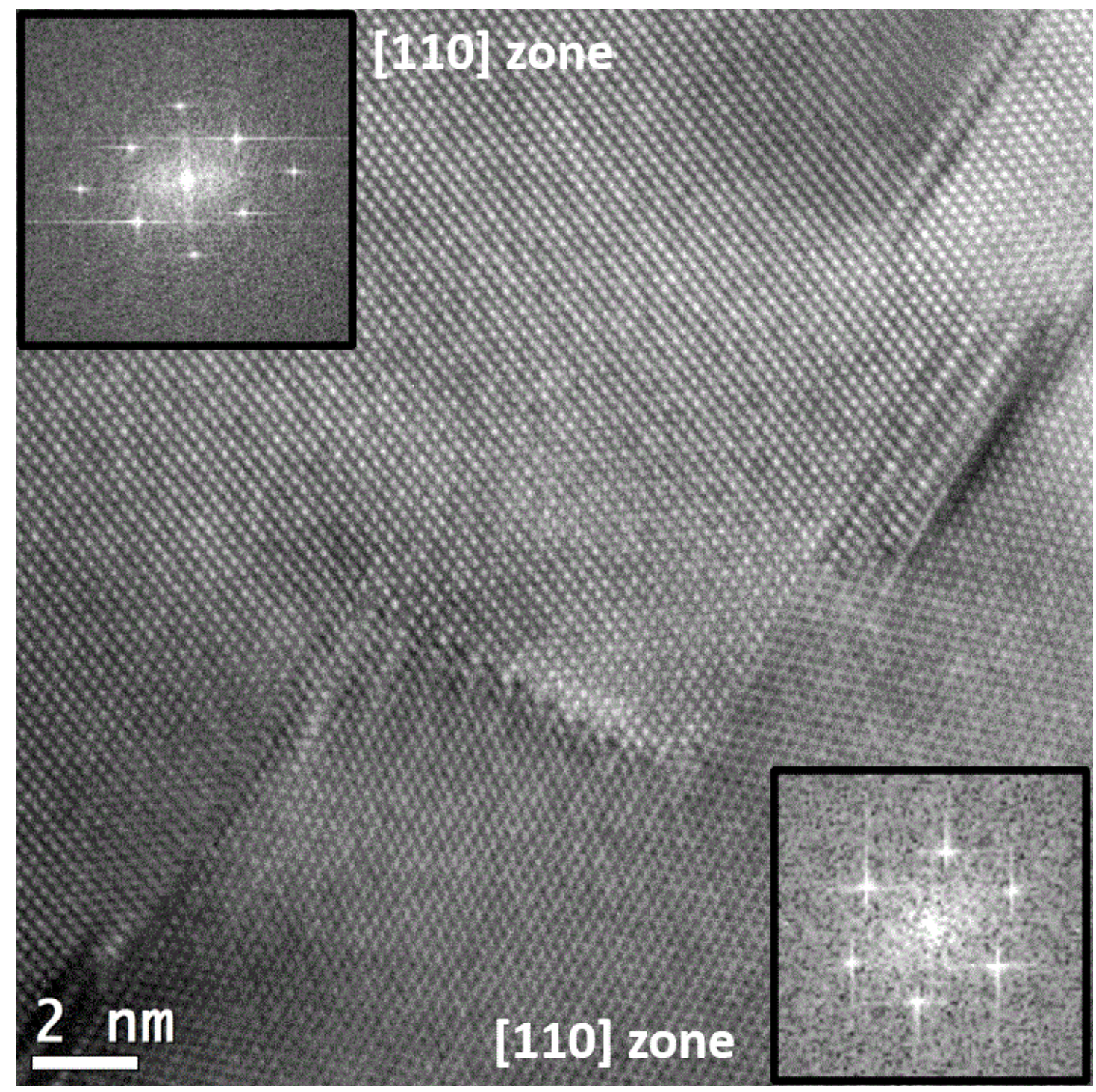

Fig. 11. High resolution image of the investigated twin boundary. The FFT patterns are indicating the exact [110] zone orientation. The same type of zones are rotated in the two grains. 


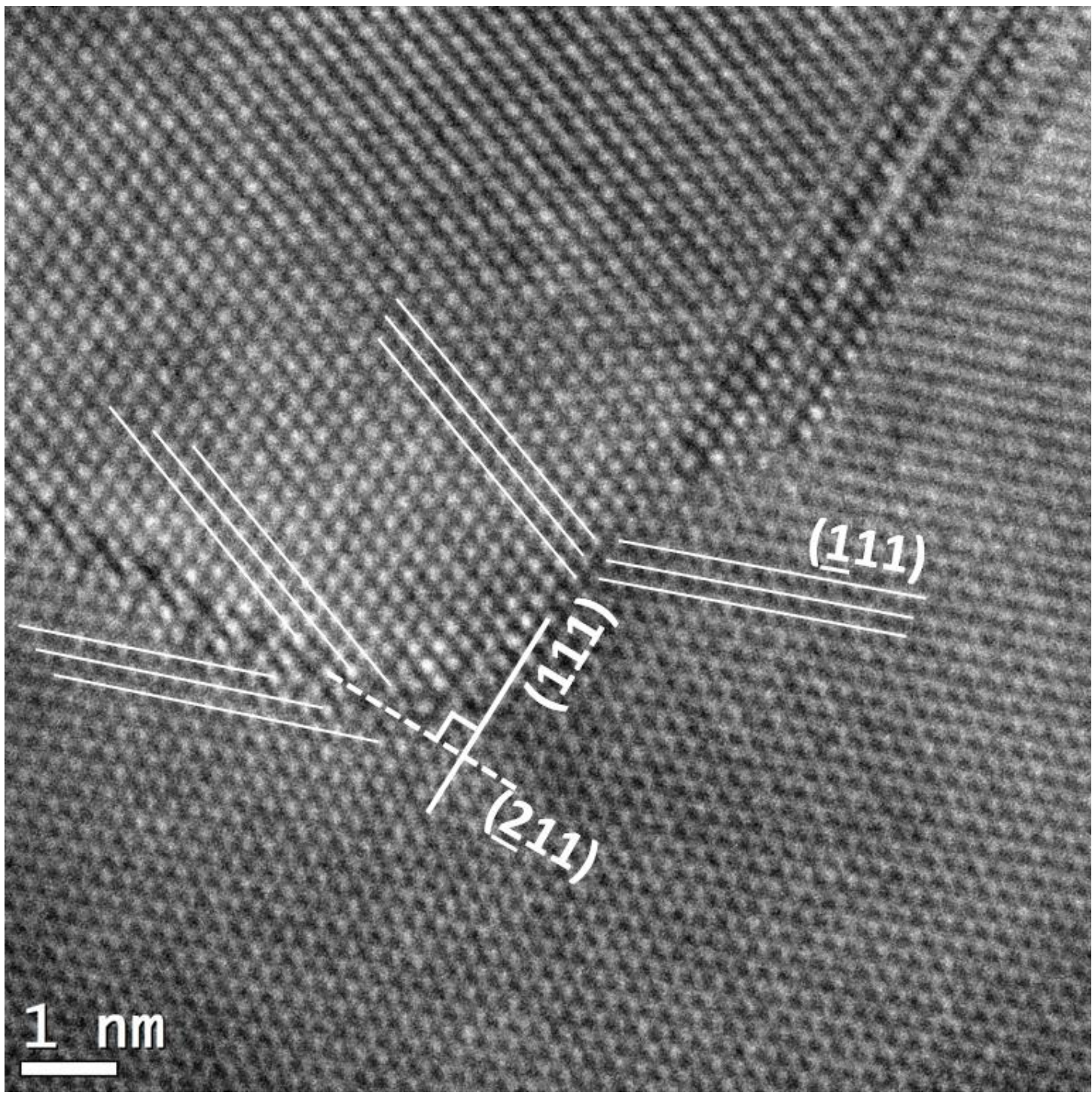

Fig. 12. High resolution image of the investigated twin boundary. (111)-plane boundary (coherent twin boundary) is marked by thick straight line, while the (211)plane boundary is marked by dashed line (incoherent twin boundary). Further (111) planes are marked by thin lines pointing out that both the coherent and incoherent twin boundaries are mirror planes with respect to the neighboring grains.

\section{Example II.}

Our second example is shown also on the Al thin film as the first one before. In the case of a less special misorientation we are still able to investigate boundaries by HR techniques, although some compromises may be introduced: one of the 
neighboring grains is imaged in a zone direction showing lattice resolution, while in the other one only one set of planes is resolved. Fig. 13/a shows the orientation map of the investigated area, in Fig. 13/b those boundaries are lighted, where the aforementioned experimental condition can be accomplished: the chosen GB is marked by an arrow where one of the neighboring grains shows lattice resolution in [110] zone and the other one shows plane resolution with (111) planes in accordance with our prediction. In the $\boldsymbol{F i g} . \mathbf{1 4} / \boldsymbol{a}_{1}$ and $\boldsymbol{F i g} . \mathbf{1 4 / \boldsymbol { b } _ { 1 }}$ CBED images are presenting the accuracy of the tilt calculations, while also the meaning of the calculated OD becomes clear: these CBED images have been taken just after the sample had been oriented in the calculated tilt position. The values of the calculated and the refined tilt positions and the OD are detailed in the Table 2. Theoretically the calculated value of the OD indicates how far a grain lies from the two-beam condition, while the other grain is set in an exact zone position. In the case of a small value of that $\left(<0.5^{\circ}\right)$, both the lattice and the plane resolution can be reached by an appropriate tilt refinement (see Fig. 14/a $\boldsymbol{a}_{2}$ and Fig. $14 / \boldsymbol{b}_{2}$ ). The high resolution image ${ }^{4}$ is shown in the Fig. 15 when the sample reached the final refined position.

\footnotetext{
${ }^{4}$ The sample is of medium thickness, where both the Kikuchi bands can be seen after contrast enhancement in the CBED and a HRTEM can also be seen, although with not the optimum quality.
} 


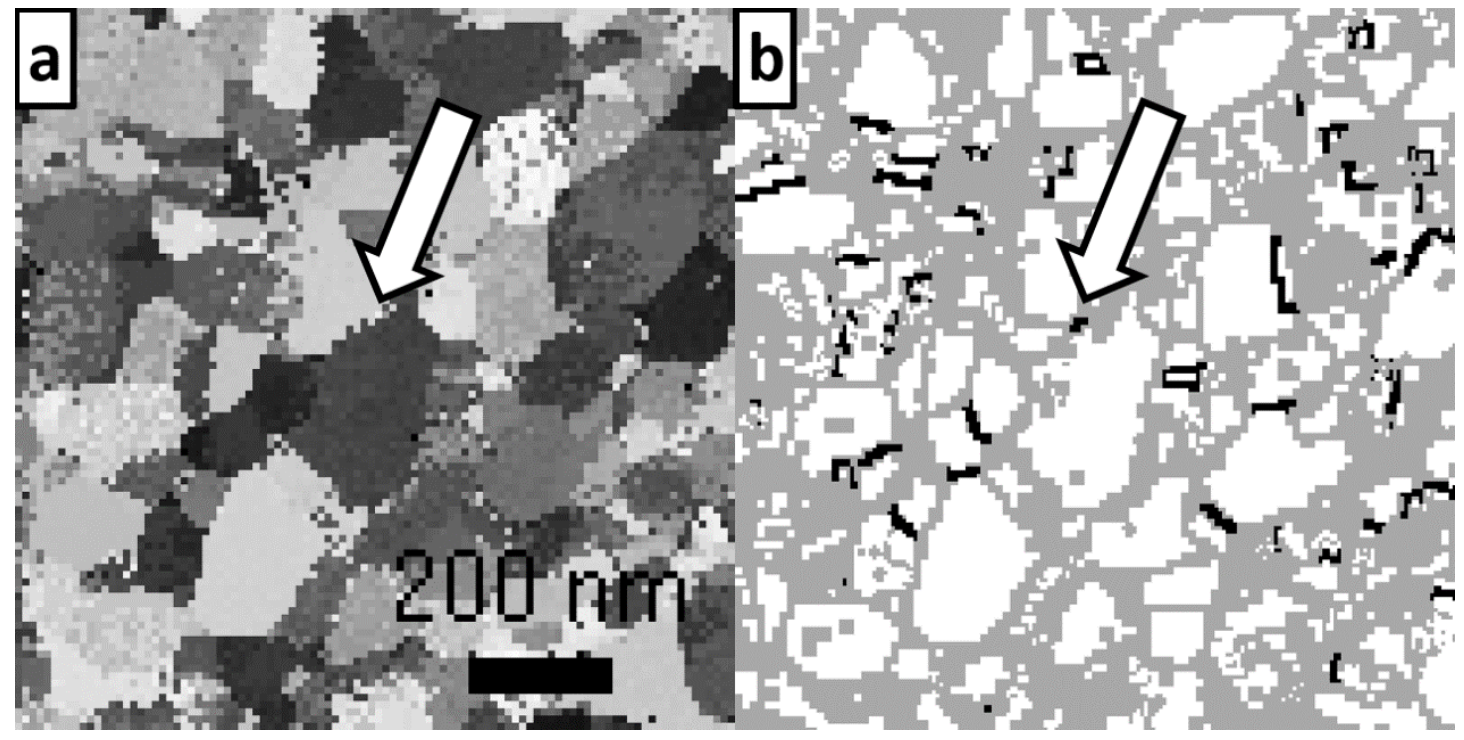

Fig. 13. Orientation map of an Al foil - the coloring displays the orientation

distribution in the X-direction (a). The black-highlighted boundaries are available for lattice and plane resolution HRTEM imaging (b). The investigated boundary is marked by an arrow. 


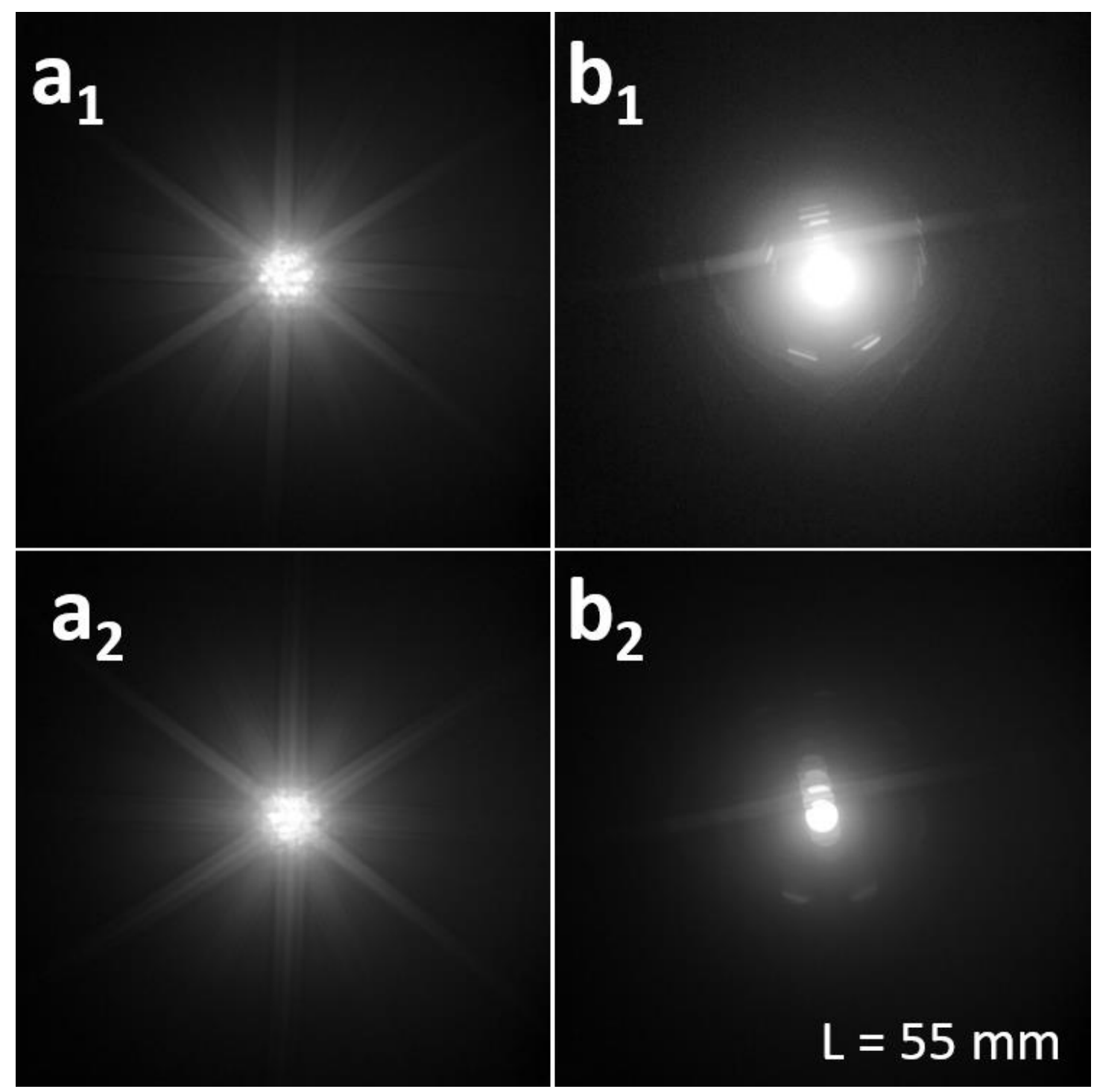

Fig. 14. CBED patterns taken on the investigated neighbors, while the sample has been oriented to the calculated tilt position: one of the neighboring grains lies in almost exact [110] zone position $\left(a_{1}\right)$, and the other one is near to a two-beam condition $\left(b_{1}\right)$. CBED patterns has been taken at the same grains after the tilt refinement ( $a_{2}$ and $\left.b_{2}\right)$.

Table 2. Experimental conditions of HTEM imaging

\begin{tabular}{|l|l|l|l|l|}
\hline Grain & Zone or & Predicted & Calculated & Refined tilt position to zone \\
\hline
\end{tabular}




\begin{tabular}{|c|r|l|l|l|}
\hline & plane to set & OD & tilt position & axis orientation in grain $\boldsymbol{a}$ \\
\hline $\boldsymbol{a}$ & {$[110]$} & \multirow{2}{*}{$0.47^{\circ}$} & $7.6^{\circ} / 7.1^{\circ}$ & $8.4^{\circ} / 7.8^{\circ}$ \\
\hline $\boldsymbol{b}$ & $(111)$ & & & \\
\hline
\end{tabular}

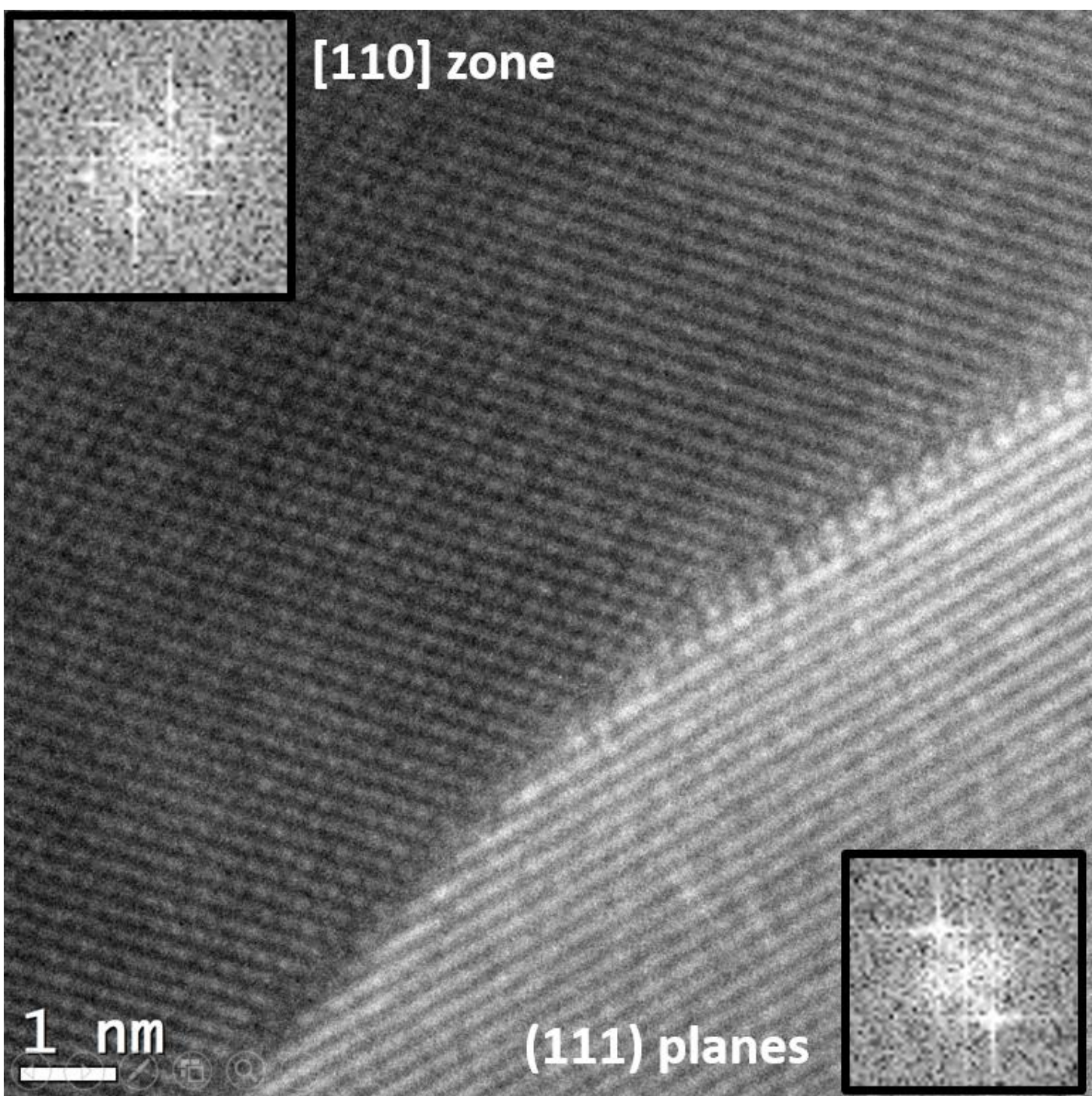

Fig. 15. High resolution image of the investigated boundary. The FFT patterns

are indicating the exact [110] zone orientation in the upper grain and the (111)

planes at the bottom. 


\section{Example III.}

Our next example is presented here on an $h c p \mathrm{ZnO}$ thin film with grain size of ca. 20-40 nm deposited on a Si substrate. Fig. 16 shows the orientation map of the selected area. Different colors represent different orientations (the green area at bottom5 is the Si substrate), therefore individual grains can be recognized. The chosen boundary is marked by the white arrow. Fig. 17 shows high resolution image of the observed boundary: one of the neighboring grains is imaged with lattice resolution from [011] i.e. [- $\left.\begin{array}{llll}-1 & 2 & -1 & 3\end{array}\right]$ zone direction, while only the (011) i.e. ( $\left.\begin{array}{llll}0 & 1 & -1 & 1\end{array}\right)$ planes are resolved in the other grain. In the area marked by the dashed rectangle no overlap is seen; so the interface plane cannot be tilted too much away from the beam direction.

\footnotetext{
${ }^{5}$ light shade in the $\mathrm{B} / \mathrm{W}$ version
} 


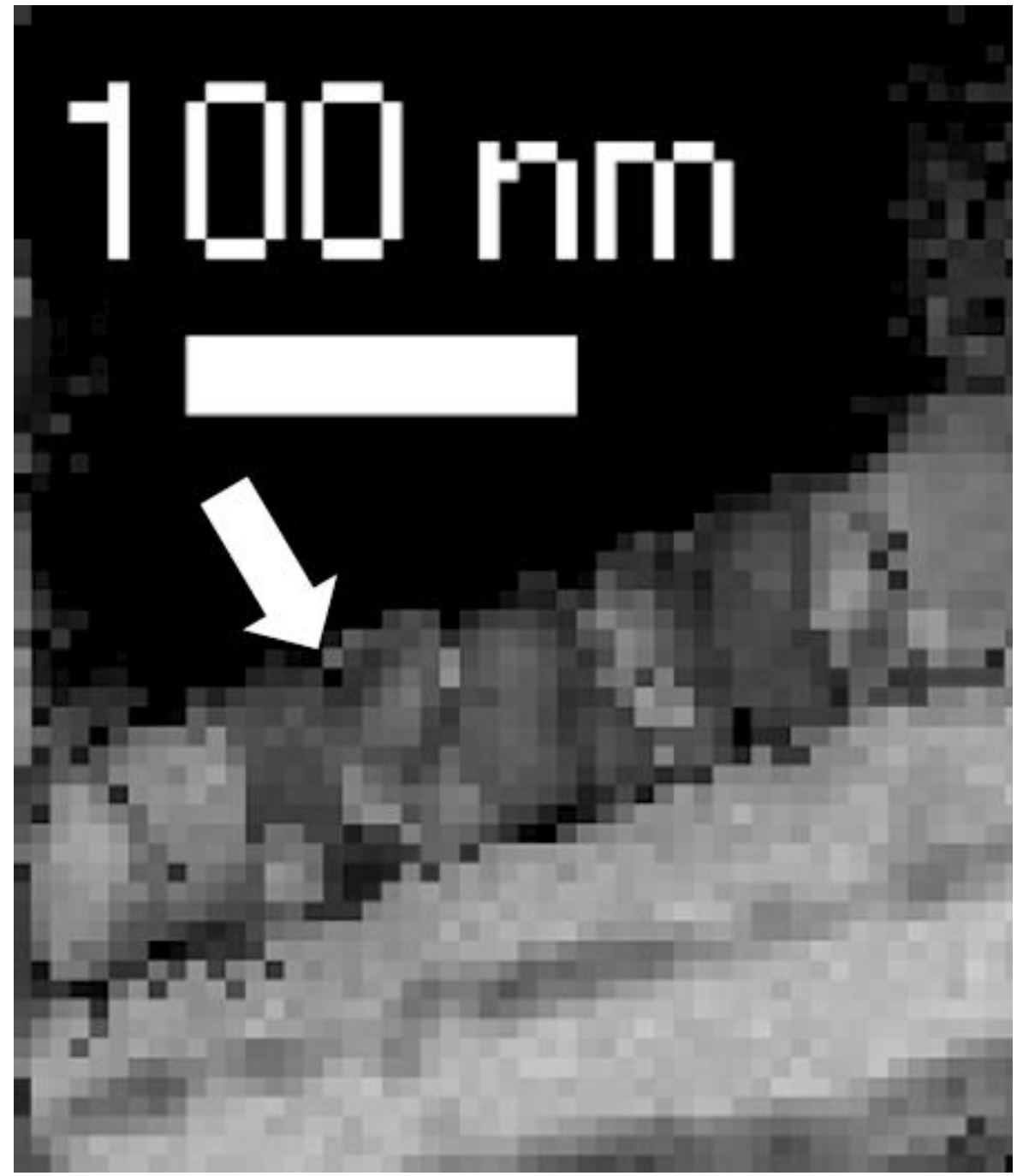

Fig. 16. Orientation map from hcp $\mathrm{ZnO}$ - orientation distribution in the Zdirection is displayed by the colors. The observed boundary is marked by the white arrow. 


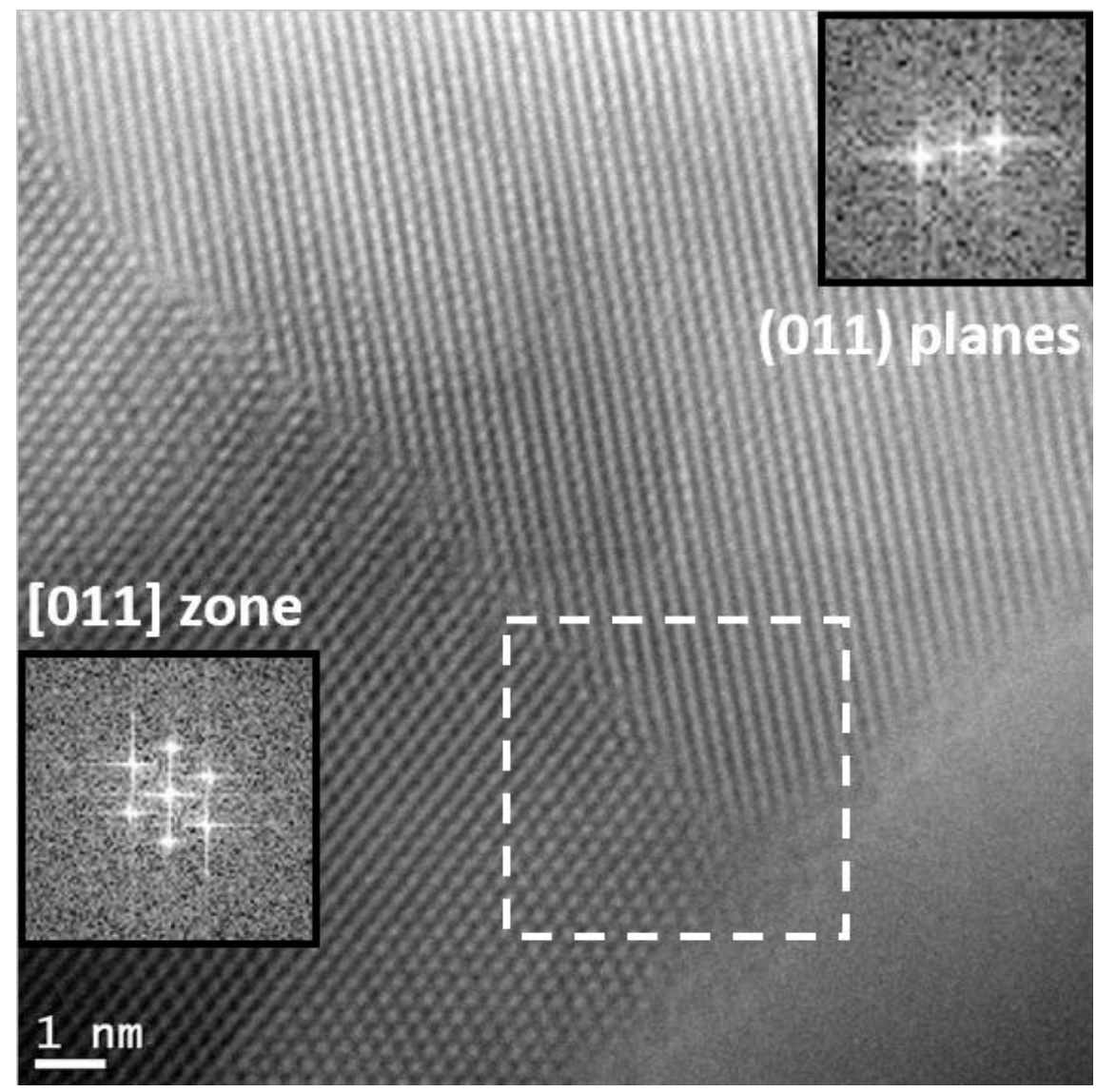

Fig. 17. The chosen boundary (from Fig. 15) shows no overlapping in the

indicated area, while the neighboring grains are imaged with lattice resolution (viewed from [011] i.e. [-1 2-1 3] zone) and plane resolution (showing the (011) i.e. (0 1 -1 1) planes).

\section{Example IV.}

In our last example the aforementioned $f c c \mathrm{Al}$ thin film has been examined again. The investigated boundary was intentionally tilted so that only one set of planes per grain are resolved, with the aim in mind to determine simultaneously both the indices of the GB plane and the thickness of the Al foil (Fig. 18). Note, that this kind of experimental condition can be achieved quite often as it is illustrated by Fig. 7, so this method of determining local thickness can be applied quite generally. As we 
mentioned before, this calculation needs at least two (but sometimes more than two) independent measurements. In the present case we chose two GBs and did two measurements per GB, each with different tilt positions. Although we got two solutions per GB, the solution common to the two pairs selects the physically relevant one resulting in $30 \mathrm{~nm}$ in thickness in the example. Note, that only the crystalline phase has been taken into account for the calculation of thickness, while the presence of the amorphous carbon supporting layer has no impact on it, in contrast to the thickness measurement based on electron energy loss spectroscopy (EELS) techniques.

Cross section of another $\mathrm{Al}$ thin film grown under identical conditions on an oxidized silicone substrate has also been investigated (Fig. 19) for checking if the implicit assumption in our calculation, namely that our layer can be regarded as a plan-parallel slab is justified. Although the Al layer grown on Si the substrate proved to be different ${ }^{6}$ in thickness, the top surface of the Al layer is flat, so our assumption seems to be justified.

The experimental details and results are shown in the Table 3. Both measurements give one pair of mathematically possible solutions. The common (within the experimental error) value is identified as the physically relevant solution to the problem.

Table 3. Experimental details in thickness and GB-plane determination. The physically relevant (=common) solution for thickness is in bold underlined.

\footnotetext{
${ }^{6}$ It is known that sticking coefficients for different substrates are different, resulting in different
} layer thicknesses even under identical deposition conditions. 


\begin{tabular}{|c|c|c|c|c|c|}
\cline { 2 - 3 } \multicolumn{2}{|c|}{ resolved planes } & \multicolumn{2}{c|}{$\begin{array}{c}\text { calculated thickness } \\
\text { values [nm] }\end{array}$} & $\begin{array}{c}\text { GB planes in the } \\
\text { neighboring } \\
\text { grains* }\end{array}$ \\
\hline \multirow{2}{*}{ GB I.** } & $1^{\text {st }}$ tilt & $(111) /(111)$ & \multirow{2}{*}{$20.4 \pm 2$} & $\underline{\mathbf{3 2 . 7} \pm \mathbf{3}}$ & (322) / (210) \\
\cline { 2 - 3 } & $2^{\text {nd }}$ tilt & $(111) /(111)$ & & & \\
\hline \multirow{2}{*}{ GB II. } & $1^{\text {st }}$ tilt & $(111) /(111)$ & & $\underline{\mathbf{2 9 . 4} \pm \mathbf{2}}$ & (211) / (432) \\
\cline { 2 - 3 } & $2^{\text {nd }}$ tilt & $(200) /(200)$ & & & \\
\hline
\end{tabular}

*rounded indices - their deviations from the calculated directions are smaller than $5^{\circ}$ **see Fig. 18
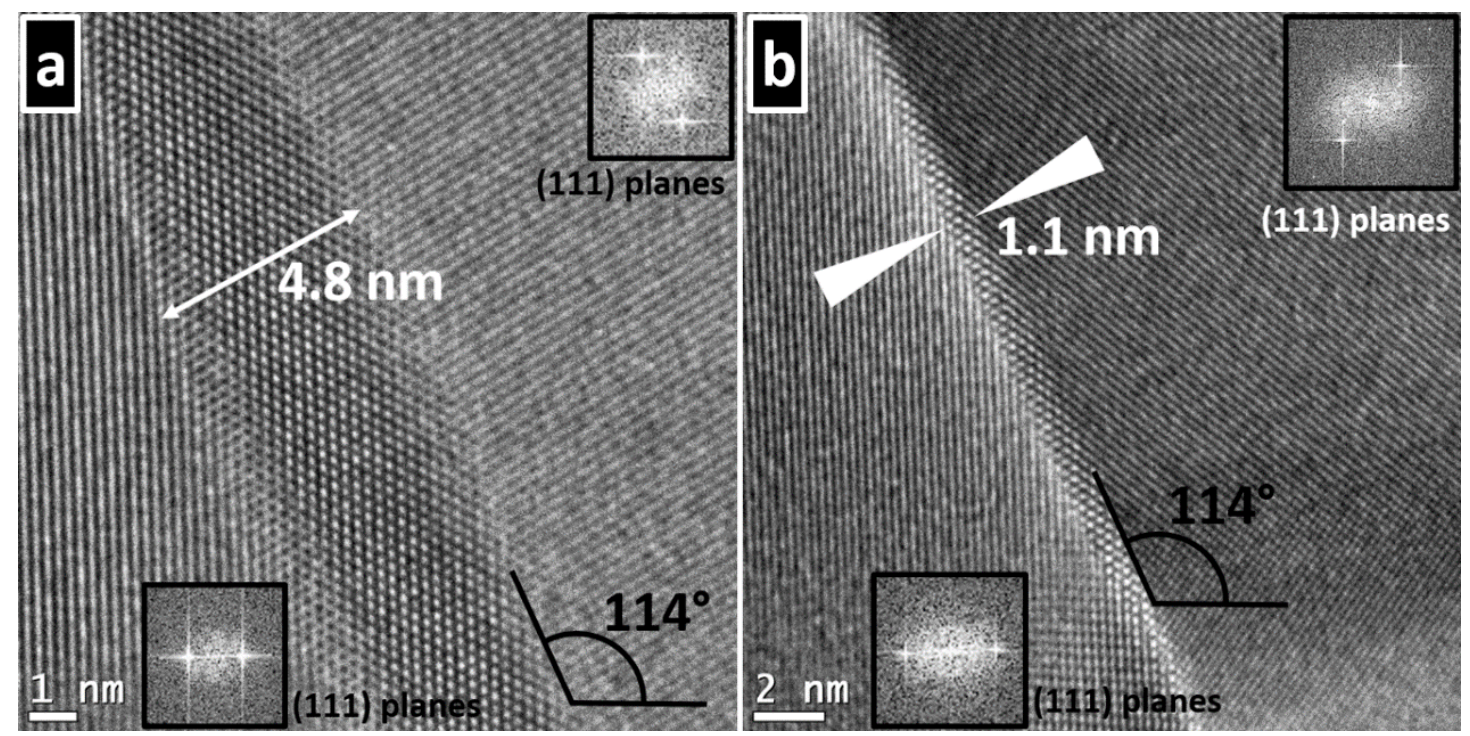

Fig. 18. High resolution images of a GB in polycrystalline Al layer taken in two different tilt positions (a), (b). In these cases the (111)-planes are resolved in both of the neighboring grains. The resulting pattern makes the overlapping area easy to characterize. 


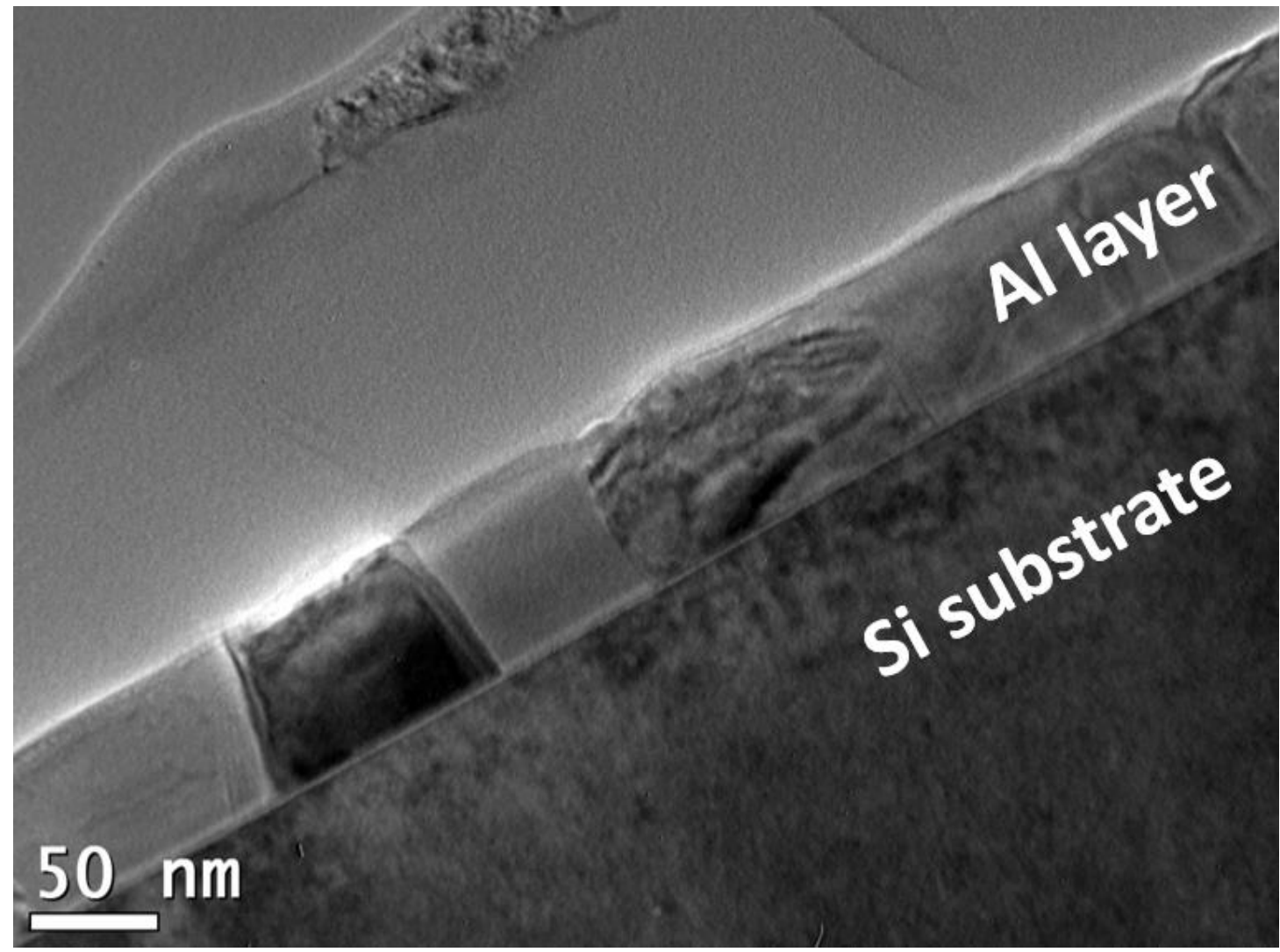

Fig. 19. Cross-section of Al layer grown in Si substrate. The thickness of the Al film seems to be homogenous. Its small fluctuations contribute to the uncertainties in the reported thickness calculation.

\section{Conclusions}

In this paper we described a method that facilitates investigating grain boundaries or phase boundaries by conventional or high resolution TEM techniques. A new approach is described here for simultaneous measurement of local thickness and indexing the grain boundary-plane both in thick and thin TEM-samples. Our method for thickness calculation is easily applicable for polycrystalline samples, while the presence of any amorphous supporting layer or contamination has no impact on the measured thickness value, in contrast to when thickness is measured by the 
EELS technique. Our software tool also helps identifying and orienting grain boundaries suitable for HRTEM examination. The other function of our software tool is to delineate the extent of grains and boundaries in noisy orientation maps. The software tool is implemented on a PC with a Windows operating system. Input to the tool is the orientation map provided by the ASTAR commercial system. Semiautomatic operation facilitates finding and examining GBs in polycrystalline thin films in a reasonable time scale. Operation is demonstrated on both cubic and noncubic crystal-systems proving that there is a reasonable chance for studying and imaging interfaces in polycrystalline samples with different crystal systems.

\section{Acknowledgment}

The authors are indebted to the NanoMegas Sprl. for providing the ASTAR system for the experiments and also for financing a one-week visit for Á Kiss to Grenoble. K. Puskás, N. Szász are acknowledged for their help in the preparation of TEM lamellae and Z. Baji for the preparation of the ZnO layer by ALD. Financial support by the Hungarian National Scientific Research Fund (OTKA) through Grant No K108869 is also acknowledged.

\section{References}

Duden, T., Gautam, A., Dahmen, U. (2011). KSpaceNavigator as a tool for computer-assisted sample tilting in high-resolution imaging, tomography and defect analysis, Ultramicroscopy $111,1574-1580$.

Edington, J. W. (1975). 2 Electron diffraction in the electron microscope, Eindhoven: N. V. Phillips' Gloeilampenfabriken 
Edington, J. W. (1976). 4 Typical electron microscope investigations, Eindhoven: N. V. Phillips' Gloeilampenfabriken

Egerton, R.F., Li, P., Malac, M. (2004). Radiation damage in the TEM and SEM, Micron 35, 399-409.

Egerton, R. F. (2011). Electron energy-loss spectroscopy in the electron microscope, Third edition, New York, Dordrecht, Heidelberg, London: Springer Science+Business Media

Forwood, C. T., Clarebrough, L. M. (1991). Electron microscopy of interfaces in metals and Alloys, p. 113. Figure 4.8. Bristol, New York: IOP Publishing

Gontard, L. C., Dunin-Borkowski, R. E., Ozkaya, D. (2008). Three-dimensional shapes and spatial distributions of $\mathrm{Pt}$ and $\mathrm{PtCr}$ catalyst nanoparticles on carbon black, Journal of Microscopy 232, 248-259

Grimmer, H., Bollmann, W., Warrington, D. H. (1974). Coincidence-site lattices and complete pattern-shift in cubic crystals, Acta Crystallographica A 30, 197207

Habas, S. E., Lee, H., Radmilovic, V., Somorjai, G. A., Yang, P. (2007). Shaping binary metal nanocrystals through epitaxial seeded growth, Nature Materials $6,692-697$.

International tables for crystallography Vol. B, second edition (2001). Edited by U. Shmueli, 3.3. Molecular modelling and graphics, p. 360. Dotrecht, Boston, London: Kluwer Academic Publishers

Jinschek, J. R., Batenburg, K. J., Calderon, H. A., Kilaas, R., Radmilovic, V., Kisielowski, C. (2008). 3-D reconstruction of the atomic positions in a simulated gold 
nanocrystal based on discrete tomography: Prospects of atomic resolution electron tomography, Ultramicroscopy 108, 589-604

Kelly, P. M., Jostsons, A., Blake, R. G., Napier, J. G. (1975). The determination of foil thickness by scanning transmission electron microscopy, Physica Status Solidi $31(2), 771-780$

Kiss, Á. K., Lábár, J. L. (2013). A method for complete characterization of the macroscopic geometry of grain boundaries; Materials Science Forum 729, 97-102.

Lábár, J. L. (2005). Consistent indexing of a (set of) single crystal SAED pattern(s) with the ProcessDiffraction program. Ultramicroscopy 103 (3), 237-249.

Lábár, J. L., Kiss, Á. K., Christiansen, S., Falk, F. (2012). Characterization of Grain Boundary Geometry in the TEM, exemplified in Si thin films; Solid State Phenomena 186, 7-12.

Loretto, M. H., Smallman, R. E. (1975). Defect analysis in electron microscopy, London: Chapman and Hall Ltd

Pozsgai, I. (1997). The determination of foil thickness by scanning transmission electron microscopy, Ultramicroscopy 68 (1), 69-75.

Qu Ying-Donga, Cui Cheng-Songa, Chen San-Benb, Li Jin-Quana (2005). A fast subpixel edge detection method using Sobel-Zernike moments operator, Image and Vision Computing 23, 11-17.

Randle, V. (1993). The Measurement of Grain Boundary Geometry, London: The Institute of Physics Publishing

Randle, V. (2001). A methodology for grain boundary plane assessment by single-section trace analysis, Scripta materiala 44, 2789-2794 
Rauch, E. F., Véron, M., Portillo, J., Bultreys, D., Maniette, Y., Nicolopoulos, S. (2008). Automatic crystal orientation and phase mapping in TEM by precession diffraction, Microscopy and Analysis 22 (6), S5-S8

Saylor, D. M., Morawiec, A., Rohrer, G. S. (2003). Distribution of grain boundaries in magnesia as a function of five macroscopic parameters, Acta Materialia $51,3663-3674$

Saylor, D. M., El-Dasher, B. S., Adams, B. L., Rohrer, G. S. (2004). Measuring the Five-Parameter Grain-Boundary Distribution from Observations of Planar Sections, Metallurgical and Materials Transactions A, 35A, 1981-1989.

Sobel, I. (1978). Neighborhood coding of binary images fast contour following and general array binary processing, Computer Graphics and Image Processing 8, $127-135$.

Spence, J. C. H., Zuo, J. M. (1992). Electron Diffraction, New York: Plenum Press, Appendix 3.7.

Stadelmann, P. A. (1987). EMS - A software package for electron diffraction analysis and HREM image simulation in materials science, Ultramicroscopy 21,131 146.

Van Aert, S., Batenburg, K. J., Rossell, M. D., Erni, R., Van Tendeloo, G. (2011). Three-dimensional atomic imaging of crystalline nanoparticles, Nature 470, $374-377$

WEB: http://www.nanomegas.com/ 